\title{
Model Reduction and Sensor Placement Methods for Finite Element Model Correlation
}

\author{
J.F. Mercer ${ }^{1}$ and G.S. Aglietti ${ }^{2}$ \\ University of Surrey, Guildford, Surrey, GU2 7XH, United Kingdom \\ and \\ A.M. Kiley ${ }^{3}$ \\ Airbus Defence and Space, Stevenage, Herts., SG1 2AS, United Kingdom
}

The issue of model reduction is one that must often be overcome in order to perform the necessary checks as part of the spacecraft Finite Element Model (FEM) validation process. This work compares different reduction methods; specifically the popular and long-standing Guyan method, and the potentially more accurate System Equivalent Reduction Expansion Process (SEREP). The influence of sensor set location on the quality of the reduced model has also been considered, and the commonly applied methods to maximize kinetic energy and effective independence have been applied. These investigations have taken the form of studies involving two large, unique, scientific spacecraft. The computational results are compared with experimental results that are also detailed in the paper. The findings highlight the potential issues with the accuracy of a Guyan reduced model in replicating the full system dynamics, even with a reasonably large sensor set. It is shown that this can be improved slightly in some circumstances through implementation of sensor set placement optimization techniques. The SEREP method is shown to have the benefit of being more accurate at replicating the full system behavior than the more traditional Guyan method, while also producing higher diagonal values in cross-orthogonality comparisons between FEM and test.

\section{Nomenclature}

\footnotetext{
$\mathrm{a}=$ candidate sensor set

$\mathbf{C}=$ damping matrix

${ }^{1}$ Research Student, Surrey Space Centre, University of Surrey

${ }^{2}$ Professor, Surrey Space Centre, University of Surrey

${ }^{3}$ Senior Specialist, Airbus Defence and Space, Stevenage
} 


$\begin{array}{lll}\mathbf{f} & = & \text { applied force vector } \\ \mathbf{I} & = & \text { identity matrix } \\ \mathbf{K} & = & \text { stiffness matrix } \\ \mathbf{m} & = & \text { master degrees of freedom (to be retained) } \\ \mathbf{M} & = & \text { mass matrix } \\ \mathbf{Q} & = & \text { Fisher independence matrix } \\ \mathbf{S} & = & \text { slave degrees of freedom (to be eliminated) } \\ \mathbf{u} & = & \text { physical displacement } \\ \boldsymbol{\eta} & = & \text { modal displacement vector } \\ \boldsymbol{\Phi} & =\text { experimental mode shape vector } \\ \boldsymbol{\Phi} & =\text { analytical mode shape vector }\end{array}$

\section{Introduction}

GRM a structural perspective, launch is one of the most challenging phases in the mission of a spacecraft. The interaction between the spacecraft and the launch vehicle is an important aspect of this; however, it is not possible to practically test the two systems coupled together. Thus, in order to simulate the launch environment, Coupled Loads Analyses (CLAs) are carried out which couple a Finite Element Model (FEM) of the spacecraft with one of the launcher to virtually predict flight loads. If there is to be confidence in the results of the CLA, it is necessary to first validate the spacecraft FEM against appropriate test measured data in order to ensure that the math model is able to reproduce accurately the behaviour of the physical hardware. A correlation process is therefore initiated, in which the analytical and experimental results are compared and the FEM updated to reconcile differences between test and analysis.

Comparisons between test and Finite Element Analysis (FEA) are made by using modal vector based metrics, such as: Modal Assurance Criteria (MAC) [1] and Cross-Orthogonality Checks (COC) [2]. Where the MAC check is a comparison between two vectors, typically the test derived mode shapes and FEA eigenvectors; and the COC check is an orthogonality check that uses the system mass matrix [3]. The use of the mass matrix serves to weight the Degree of Freedom (DoF) importance based on modal mass, which is not accounted for by the basic MAC check. 
The use of the orthogonality metric does, however, introduce the issue of the order of the mass matrix. When computing the MAC, vectors describing the mode shapes must be of equal length. The FEM is likely to have data for hundreds of thousands, if not millions, of DoFs, compared to data for only a few hundred DoFs captured by accelerometers during the test. MAC can nonetheless be used by simply partitioning out the FEM eigenvector values for DoFs corresponding to the test measurement point plan (MPP), and, therefore, does not require further manipulation of the model. For the orthogonality checks, the mass matrix dimensions must match the modal vector order. It is therefore necessary to either expand the experimental data to a DoF count matching the FEM or reduce the analytical results to the DoFs corresponding to the test accelerometers [4]. A potential problem with using the FEM to expand the test data is that any errors in the FEM, which is still to be validated, may corrupt the experimental data and undermine the following correlation and update process. It is therefore generally considered that the modal reduction of the FEM to the test measured DoFs, to create a Test Analysis Model (TAM) of the structure, is the preferred approach [5].

There are many methods currently available for performing these reductions. There have already been several studies [6-12] comparing the various methods including; Static (or Guyan) reduction [13], Improved Reduction System (IRS) [14], Dynamic reduction [15], Modal reduction [5], System Equivalent Reduction Expansion Process (SEREP) [16], Hybrid [17] and Craig-Bampton [18]. Here the focus is on: the Guyan reduction method, which has some historical basis and availability within FE tools such as MSC-NASTRAN [19]; and SEREP which has been identified as potentially more suitable for generating spacecraft TAMs $[20,21]$. Where most previous work has focused on small, generic example cases, the main contribution of this work is to show and identify how these methodologies perform with real spacecraft and typical FEMs generated in industry. Another aspect to be considered by this work is the influence of different sensor placement options on the respective reduction methods.

The choice of test sensor/accelerometer set location MPP is crucial in order to sufficiently capture the dynamics of the system. Good quality test data is vital if the resulting spacecraft FEM validation is to be meaningful. The experimental modal parameters used in the validation of spacecraft FEMs are typically estimated from data collected during fixed-base sine-sweep testing performed through use of an electro-dynamic shaker. In specifying the MPP in preparation for a spacecraft sine test, usually the first point of focus is capturing response measurements at all unit or equipment mounting locations, key sub-system locations (often prescribed as suitable monitor locations by subsystem contractors) and CLA recovery locations. Such primary focus on data recovery, in terms of obtaining inputs 
into the payload or responses on the payload, often accounts for a substantial portion of available channel count which can leave a potential smaller subset available whose purpose is primarily to support modal correlation. All modes of significance, both to the global structure and to any critical local modes on sub-systems, must be captured with sufficient signal strength and have independence such that each mode is distinct and easily identified and differentiated from the other modes of the system. In addition, the spacecraft FEM correlation process requires the production of a good quality reduced TAM to increase the likelihood of achieving acceptable results for the COC. The quality of the final TAM depends heavily on the reduction method being employed and also on the retained DoFs of the system. As such pre-test methods to improve the placement of sensors have been created [22-26].

Over the years, various sensor placement optimization methods have been developed which employ the FEM to identify a MPP set pre-test, such that the data collected is of high quality for clear mode identification and also for production of accurate, robust TAMs. Most methods involve the identification of the 'best' degrees of freedom (DoFs) to keep from an initial larger candidate set, however, more recently some methods have been developed which expand out from a small initial set to a larger final set [27]. Both iterative methods, such as the Effective Independence (EfI) method [28], and single calculation methods, such as modal kinetic energy (KE) [29], are available. Sensors may be considered individually as separate DoFs, or in grouped form such as in the case of triaxial sensors [30].

Flanigan [10] observed that certain reduction methods appeared to be more influenced than others by an inadequate sensor set. Bergman et al [6] also explored different reduction methods, and proposed that many previous studies, such as those by Chung [8], Freed and Flanigan [9], and Avitabile et al [11], had failed to account for the fact that different reduction methods require different sensor placement optimization methods. Most previous work exploring reduction methods had taken a single sensor set, determined from a particular sensor placement optimization method, and had compared the reduction methods for that particular set. As such, Bergman et al [6] attempted to compensate for this by applying different sensor placement methods as appropriate for each reduction method, in order to assess the quality and robustness of the resulting TAMs with respect to the same original full FEM. Of the sensor placement methods considered, SEREP performed best when EfI [27] was applied to ensure optimum linear independence of target modes, whereas for static reductions, a KE based technique was employed. It is therefore clear that the sensor placement method must be consistent with the reduction method to be employed, as the two are interconnected. As such, this work takes a similar approach in that both EfI and KE based 
sensor placement techniques are explored. In addition to these optimized sensor placements, as considered in the works previously cited, this work also considers the real MPPs used in spacecraft testing, which account for practical considerations of accessibility and areas of interest to predict load levels around delicate equipment. Where the Bergman et al study [6] focused on one simple, generic satellite FEM with fewer than ten thousand DoFs in total; this work considers two real, large, scientific spacecraft (BepiColombo and Aeolus) FEMs with complex architectures and DoF counts in the order of hundreds of thousands. The use of real spacecraft in this study has enabled final comparisons to be made between the FEM and the test mode shapes. While previous work has investigated the robustness of reduction methods in theory using analytical models [21], the differences between test and FEM mode shapes can be caused by a wide variety of factors and as such can be difficult to replicate using purely mathematical analyses. In real testing it is possible that: not all vibration modes will be properly excited; the dynamic influence of coupling with the shaker structure can influence the responses; there can be additional uncertainty in the test mode shapes introduced through curve-fitting estimations. While the large, complex FEM may not match the test due to a variety of issues, such as incorrect modelling assumptions in the rigid constraints applied at the base. The comparison with test data presented here allows for a realistic assessment of the suitability of the reduction methods to create reduced TAMs for actual test-FEM correlation, rather than more 'academic' studies; the conditions of which often minimize or omit many of these factors. The fact that two real spacecraft have been considered gives wider relevance to the results of this work.

The studies presented herein are focused on the issues associated with the placement of accelerometers during modal testing and the subsequent reduction of the mathematical model to the corresponding DoFs. The need to perform such activities is a direct consequence of the use of discrete measurement methods (i.e. only having data at a limited number of selected discrete points on the test structure for comparison with the mathematical model). Many of the most established model reduction methods have been around for decades [31]. During that time, the number of accelerometers used on typical modal vibration tests has not increased in line with the increase in FEM 'size', with FEMs comprising of over a million DoFs now common in many applications (such as those considered in this paper). This increased mismatch in the amount of data from test and FEM has exacerbated the issues associated with model reduction, and as such this work presents the results of reductions performed on larger, more complex FEMs than many of those considered in older studies or those focused on smaller/simpler academic example cases. 
Full field measurement methods and those able to capture data at more numerous points have been developed which may address some of the issues associated with the traditional discrete measurement techniques. These include the application of non-contact approaches such as digital image correlation (DIC) [32, 33] and the use of Laser Doppler Vibrometers (LDVs) [34, 35]. The non-contact measurements will have the additional benefit of not mass-loading the structure, which can be an issue with traditional methods employing sensors attached to the structure. The availability of full field data may also enhance the ability to identify and distinguish subtleties in different vibration mode shapes from the test in cases where this may not have been achieved with a discrete MPP. Of the aforementioned optical measurement techniques, the use of LDVs is more common for vibration testing at present, however issues including the expense and time required have so far limited the range of applications [31]. The development of experimental modal analysis methods employing DIC in 3-dimensions has shown feasibility, but the technology remains too immature [31] to be considered for industrial applications such as those addressed herein. Research into this subject is ongoing with several studies comparing the more traditional modal testing methods, such as roving hammer tests using accelerometers, with these non-contact optical approaches [31, 36] as well as work being undertaken to develop the new data processing and correlation methods necessary to account for large amounts of data gathered from the full field of interest [37-39].

Additionally, the field of strain modal analysis may become more wide-spread in years to come. The use of distributed fibre optic strain sensors has become common in structural health monitoring [40]; however, their use may be broadened into modal testing applications as developments, such as Fiber Bragg Grating sensors [41], continue overcome some of the issues which had previously inhibited the practicality of strain gauges versus the more easily applied accelerometers [42].

It is possible that the future of modal testing will lie in the use of the aforementioned methods. If full-field techniques become viable for large, complex structures, this may circumvent many of the issues concerning sensor placement and model reductions which are presented herein. Until such time as the cost and practicality issues associated with the full field approaches are resolved however, there is still value in pursuing the best sensor placement and model reduction methods for current discrete measurement applications. 


\section{Theory}

\section{A. Modal Correlation Checks}

MAC is a simple means to determine the level of similarity between two vectors of equal order. Typically in FEM correlations, the test mode shapes and FEM eigenvectors are compared, and the MAC check yields a value between 0 and 1 which indicates how closely matched the vectors are, with 1 indicating a perfect match:

$$
\operatorname{MAC}=\frac{\left(\Psi^{\mathrm{T}} \boldsymbol{\phi}\right)^{2}}{\left(\boldsymbol{\Psi}^{\mathrm{T}} \boldsymbol{\Psi}\right) \cdot\left(\boldsymbol{\phi}^{\mathrm{T}} \boldsymbol{\phi}\right)}
$$

The experimental and analytical mode shapes are given here by $\psi$ and $\varphi$ respectively.

It is generally considered, and indicated in European ECSS standards [43] and similar NASA guidelines [44], that target modes achieving a MAC of at least 0.9 indicates a good correlation and is the target value for the fundamental modes of a spacecraft.

The COC is also an ECSS [43] required check, and works similarly to MAC, but with the mass matrix employed to weight the relative importance of the DoFs being considered. An ideal result of perfectly matched mode shapes which are orthogonal to the mass matrix will yield a diagonal matrix, and for mass normalized modes this becomes an identity matrix. In the ESA and NASA standards [43, 44], it is specified that off-diagonal values $<0.1$ and leading diagonal terms $>0.9$ are deemed to indicate a good level of correlation. The typical and normalized (NCO) forms of the COC are given by:

$$
\begin{gathered}
\mathrm{COC}=\frac{\left(\boldsymbol{\Psi}^{\mathrm{T}} \mathbf{M}_{\mathrm{TAM}} \boldsymbol{\phi}\right)}{\sqrt{\left(\boldsymbol{\Psi}^{\mathrm{T}} \mathbf{M}_{\mathrm{TAM}} \boldsymbol{\psi}\right)} \sqrt{\left(\boldsymbol{\phi}^{\mathrm{T}} \mathbf{M}_{\mathrm{TAM}} \boldsymbol{\phi}\right)}} \\
\mathrm{NCO}=\frac{\left(\boldsymbol{\Psi}^{\mathrm{T}} \mathbf{M}_{\mathrm{TAM}} \boldsymbol{\phi}\right)^{2}}{\left(\boldsymbol{\Psi}^{\mathrm{T}} \mathbf{M}_{\mathrm{TAM}} \boldsymbol{\Psi}\right)\left(\boldsymbol{\Phi}^{\mathrm{T}} \mathbf{M}_{\mathrm{TAM}} \boldsymbol{\phi}\right)}
\end{gathered}
$$

where the TAM, or reduced mass matrix, $\mathrm{M}_{\mathrm{TAM}}$, is generated by application of an appropriate model reduction method. The experimental and analytical mode shapes are again given by $\psi$ and $\varphi$ respectively.

\section{B. Model Reduction Methods}

\section{Guyan Reduction}

Guyan reduction has been considered for investigation as it is one of the most commonly used reduction methods in industry. Due to its popularity, this reduction method is supported by many of the widely used FEA software 
packages; for example, this work employs standard MSC-NASTRAN [19] alters to perform the reductions of the spacecraft models.

Guyan reduction is a static reduction method and, therefore, is not able to accurately capture the exact dynamics of the full system. Flanigan [10] showed that Guyan reduction produces a TAM which does not represent the full system as accurately as more sophisticated methods, such as IRS and Dynamic reduction, which had been developed subsequently to overcome the shortcomings of static methods. One reason for its continued popularity, despite its limitations, is that Guyan reduction is simple to perform and is an inherent production routine within certain FE codes. Another key advantage is that the resulting TAM also has been shown, e.g. by Chung and Simonian [8], to have the benefit of being relatively robust, compared to modal methods such as SEREP, at achieving low offdiagonal COC results in spite of noise and small errors in the test or FEM.

The dynamics of a system can be defined by the following equation:

$$
\mathbf{M u}+\mathbf{C u}+\mathbf{K u}=\mathbf{f}
$$

where $\mathbf{M}, \mathbf{C}$ and $\mathbf{K}$ are the mass, damping and stiffness matrices respectively, $\mathbf{u}$ is the physical displacements and $\mathbf{f}$ is the applied forces.

In order to perform the Guyan, or static, reduction equation (4) can be written in the following, partitioned form, with the damping neglected:

$$
\left[\begin{array}{cc}
\mathbf{M}_{\mathrm{mm}} & \mathbf{M}_{\mathrm{ms}} \\
\mathbf{M}_{\mathrm{sm}} & \mathbf{M}_{\mathrm{ss}}
\end{array}\right] \cdot\left\{\begin{array}{c}
\ddot{\mathbf{u}}_{\mathrm{m}} \\
\ddot{\mathbf{u}}_{\mathrm{s}}
\end{array}\right\}+\left[\begin{array}{cc}
\mathbf{K}_{\mathrm{mm}} & \mathbf{K}_{\mathrm{ms}} \\
\mathbf{K}_{\mathrm{sm}} & \mathbf{K}_{\mathrm{ss}}
\end{array}\right] \cdot\left\{\begin{array}{c}
\mathbf{u}_{\mathrm{m}} \\
\mathbf{u}_{\mathrm{s}}
\end{array}\right\}=\left\{\begin{array}{c}
\mathbf{f}_{\mathrm{m}} \\
\mathbf{0}
\end{array}\right\}
$$

Here the subscripts $m$ and $s$ are used for the master and slave DoFs respectively, where the masters are the DoFs of interest to be retained and the slaves are those to be eliminated in the reduction. In an NASTRAN context, the "masters" may be considered the problem A-Set definition in NASTRAN terminology.

For the static reduction, the inertias of the slave DoFs are assumed small and thus are neglected, allowing the second line of equation (5) to be simplified to:

$$
\mathbf{K}_{\mathrm{sm}} \mathbf{u}_{\mathrm{m}}+\mathbf{K}_{\mathrm{ss}} \mathbf{u}_{\mathrm{s}} \approx \mathbf{0}
$$

This allows for the slave displacements to be defined in terms of the master displacements, and this transformation may then be substituted back into equation (5) as follows:

$$
\left[\begin{array}{cc}
\mathbf{M}_{\mathrm{mm}} & \mathbf{M}_{\mathrm{ms}} \\
\mathbf{M}_{\mathrm{sm}} & \mathbf{M}_{\mathrm{ss}}
\end{array}\right]\left[\begin{array}{c}
\mathbf{I} \\
-\mathbf{K}_{\mathrm{ss}}^{-1} \mathbf{K}_{\mathrm{sm}}
\end{array}\right] \cdot\left\{\ddot{\mathbf{u}}_{\mathrm{m}}\right\}+\left[\begin{array}{cc}
\mathbf{K}_{\mathrm{mm}} & \mathbf{K}_{\mathrm{ms}} \\
\mathbf{K}_{\mathrm{sm}} & \mathbf{K}_{\mathrm{ss}}
\end{array}\right]\left[\begin{array}{c}
\mathbf{I} \\
-\mathbf{K}_{\mathrm{ss}}^{-1} \mathbf{K}_{\mathrm{sm}}
\end{array}\right] \cdot\left\{\mathbf{u}_{\mathrm{m}}\right\} \approx\left\{\mathbf{f}_{\mathrm{m}}\right\}
$$


The slave displacements, $\mathbf{u}_{\mathrm{s}}$, have been eliminated from the equation. The reduced TAM can now be defined by premultiplying by the transformation matrix, and the reduced mass matrix becomes:

$$
\left[\begin{array}{c}
\mathbf{I} \\
-\mathbf{K}_{\mathrm{ss}}^{-1} \mathbf{K}_{\mathrm{sm}}
\end{array}\right]^{\mathrm{T}}\left[\begin{array}{cc}
\mathbf{M}_{\mathrm{mm}} & \mathbf{M}_{\mathrm{ms}} \\
\mathbf{M}_{\mathrm{sm}} & \mathbf{M}_{\mathrm{ss}}
\end{array}\right]\left[\begin{array}{c}
\mathbf{I} \\
-\mathbf{K}_{\mathrm{ss}}^{-1} \mathbf{K}_{\mathrm{sm}}
\end{array}\right] \approx \mathbf{M}_{\mathrm{TAM}_{-} G u y a n}
$$

It is important to note that this method gives exact results for static problems. As the solution is derived only from the static stiffness of the system, there will be discrepancies when this is applied to dynamic analyses. As such, the Guyan reduced model will not give an exact match to the eigenvalues and eigenvectors of the full system. This means that even with an initial full FEM which sufficiently captures the dynamics of the test structure, the reduced model may produce natural frequencies and mode shapes which are different from those in the original FEM and result in non-compliance with correlation checks, such as COC. The level of imprecision is associated with the neglected inertia of the DoFs not included in the reduction. The method therefore typically becomes less accurate with increasing natural frequency of considered modes. As the quality of the TAM is heavily dependent on the omitted and selected DoFs, the choice of MPP can significantly affect the accuracy of the final result.

\section{System Equivalent Reduction Expansion Process (SEREP)}

This work has also considered SEREP reduction [16] as a potential replacement for the more commonly used Guyan technique for the reduction of spacecraft FEMs as it has been identified previously by Aglietti et al [20] as a potentially more suitable method. The main benefit of SEREP is that it computes reduced model that matches the eigenvalues and eigenvectors of the full model for the considered DoFs. This approach was originally proposed by Kammer, with the development of the Modal reduction method [5]. The term SEREP was derived when an adaptation of the original Modal reduction was developed by O'Callaghan [16]. The SEREP method presents a significant advantage as the reduced model gives a true representation of the full model dynamics, therefore providing a potentially more meaningful comparison to the test structure dynamic behavior. It is, however, important to also consider that these benefits may be lessened if the TAM is not robust for dealing with noise and errors in the test and/or FEM results, which can make it more difficult to achieve the required levels of correlation as defined by the COC checks [9]. Further investigations into the use of SEREP for spacecraft FEM reduction, such as those of Aglietti et al [20,21], have found that the robustness of the TAM to errors is dependent on the number of modes included in the reduction. It is proposed that the robustness may be improved by inclusion of only the target modes in the reduction.

The SEREP reduction method is derived by first defining the displacement of master DoFs as follows: 


$$
\mathbf{u}_{\mathrm{m}}=\boldsymbol{\phi}_{\mathrm{m}} \boldsymbol{\eta}
$$

where the target mode shapes, partitioned to the required DoFs, are contained in the modal matrix, $\boldsymbol{\phi}_{\mathrm{m}}$, which is multiplied by appropriate modal coordinates contained in the vector $\eta$.

It is then possible to rearrange the above equation:

$$
\boldsymbol{\eta}=\boldsymbol{\phi}_{\mathrm{m}}^{-1} \mathbf{u}_{\mathrm{m}}
$$

Often the number of master DoFs is significantly greater than the number of modes being considered, the following procedure can be performed to obtain the pseudo inverse of the modal matrix:

$$
\boldsymbol{\eta}=\left(\boldsymbol{\phi}_{\mathrm{m}}^{\mathrm{T}} \boldsymbol{\phi}_{\mathrm{m}}\right)^{-1} \boldsymbol{\phi}_{\mathrm{m}}^{\mathrm{T}} \mathbf{u}_{\mathrm{m}}=\boldsymbol{\phi}_{\mathrm{m}}^{\mathrm{P}} \mathbf{u}_{\mathrm{m}}
$$

where the superscript $\mathrm{P}$ represents the pseudo-inverse of the reduced modal matrix. It is therefore possible to redefine the full displacement vector, $\mathbf{u}$, as:

$$
\mathbf{u}=\boldsymbol{\phi} \boldsymbol{\eta}=\boldsymbol{\phi} \boldsymbol{\phi}_{\mathrm{m}}^{\mathrm{P}} \mathbf{u}_{\mathrm{m}}
$$

If equation (12) is substituted into equation (4), and the resulting equation is then pre-multiplied by the transpose of the reduced modal matrix and its pseudo-inverse, the result is:

$$
\boldsymbol{\phi}_{\mathrm{M}}^{\mathrm{P}^{\mathrm{T}}} \boldsymbol{\phi}^{\mathrm{T}} \mathbf{M} \boldsymbol{\phi} \boldsymbol{\phi}_{\mathrm{M}}^{\mathrm{P}} \ddot{\mathbf{u}}_{\mathrm{M}}+\boldsymbol{\phi}_{\mathrm{M}}^{\mathrm{P}^{\mathrm{T}}} \boldsymbol{\phi}^{\mathrm{T}} \mathbf{K} \boldsymbol{\phi} \boldsymbol{\phi}_{\mathrm{M}}^{\mathrm{P}} \mathbf{u}_{\mathrm{M}}=\boldsymbol{\phi}_{\mathrm{M}}^{\mathrm{P}^{\mathrm{T}}} \boldsymbol{\phi}^{\mathrm{T}} \mathbf{f}
$$

With the mode shapes mass-normalized, which is commonly performed automatically by FE software, the following holds true:

$$
\boldsymbol{\phi}^{\mathrm{T}} \mathbf{M} \boldsymbol{\phi}=\mathbf{I}
$$

Looking back to equation (13) it can be seen that the TAM can be represented in the following manner and is therefore determined from the mode shapes alone:

$$
\mathbf{M}_{\text {TAM_SEREP }}=\boldsymbol{\phi}_{\mathrm{M}}^{\mathrm{P}^{\mathrm{T}}} \boldsymbol{\phi}_{\mathrm{M}}^{\mathrm{P}}
$$

Note that no manipulation of the full FEM mass matrix is needed to obtain $\mathrm{M}_{\text {TAM_SEREP. }}$ If required, the reduced stiffness matrix may be derived in the same way. It should be noted that this method of reduction inherently yields modes exactly matching the partitioned full FEM modes.

\section{Sensor Placement Optimization Methods}

\section{Modal Kinetic Energy Method}

The concept of using energy distribution as a master DoF selection indicator has been in use for decades[45]. This led to the now commonly applied modal KE method $[29,46]$ of sensor placement selection; a computationally 
efficient single calculation method. This method estimates the dynamic contribution of the considered DoFs through use of mass and modal displacement information. This is calculated as follows:

$$
\mathrm{KE}_{\mathrm{ik}}=\phi_{\mathrm{ik}} \sum_{\mathrm{j}} \mathrm{M}_{\mathrm{ij}} \phi_{\mathrm{jk}}
$$

where i denotes the DoF index; $\mathrm{j}$ the column of the mass matrix, $\mathbf{M}$; and $\mathrm{k}$ the target mode number.

This KE calculation may be applied for all of the target modes in order to determine the DoFs associated maximized KE for the mode under consideration [6]. An appropriate number of DoFs for the final sensor set may then be selected on the basis of the associated KE.

The KE method is therefore a means to improve target mode signal strength. One limitation of this method, however, is that the linear independence of the target modes, an important factor to aid in mode identification and, therefore, test-analysis correlation is not considered. Nevertheless, this technique is still commonly used to improve the accuracy in static TAMs. Furthermore, this technique is often used as an initial method to reduce an extremely large sensor set down to a more reasonable starting point to then apply another sensor set optimization method, such as an effective independence based approach.

\section{Effective Independence Method}

In order to address the issue of linear independence, as well as signal strength, for the target modes, Kammer at al [28] developed an iterative method of sensor set selection known as the effective independence method, EfI. Other methods had previously attempted to address this, but most employed search techniques which required greater computational time and effort [28].

The EfI method takes inspiration from earlier work of Qureshi et al [47] by proposing to solve the problem of selecting a sensor set that provides the best numerical conditioning of the Fisher information matrix, $\mathbf{Q}$. The method is based on the idea that the linear independence between modes is optimized by selecting DoFs to maximize the determinant of the Fisher information matrix. The Effective Independence Matrix can be calculated as follows:

$$
\mathrm{EfI}_{\mathrm{ii}}=\left(\phi_{\mathrm{a}}\right)_{\mathrm{i}} \mathbf{Q}^{-1}\left(\phi_{\mathrm{a}}\right)_{\mathrm{i}}^{\mathrm{T}}
$$

where

$$
\mathbf{Q}=\boldsymbol{\phi}_{\mathrm{a}}^{\mathrm{T}} \boldsymbol{\phi}_{\mathrm{a}}
$$

Where $\boldsymbol{\phi}_{\mathrm{a}}$ is the modal matrix, with rows corresponding to the candidate set DoFs and columns representing the modes of interest to be found by modal analysis of the full FEM. The leading diagonal values are then $0 \leq \operatorname{EfI}_{\mathrm{i}, \mathrm{i}} \leq 1$. The lower the value of the leading diagonal, the less the corresponding DoF contributes to the independence of the 
mode shapes. Therefore, this method works by eliminating the DoFs with lowest EfI. The technique is generally applied iteratively, and the ideal case has only one single DoF eliminated in each iteration. This enables the method to account for cases where the elimination of one DoF changes the order of importance of the remaining DoFs. This process is repeated until the desired number of sensors is determined.

This method, and its variations, are commonly applied and recommended in many texts [48-50] as an appropriate method to apply when selecting sensor locations.

\section{Example Applications}

\section{A. Overview of Investigations Undertaken}

The studies presented herein focus on two large, unique, scientific spacecraft:

- The ESA/JAXA collaboration spacecraft BepiColombo for the exploration of Mercury which has a stacked configuration comprising of two planetary orbiters and a propulsion module. The spacecraft has a mass of $6446 \mathrm{~kg}$ and the FEM consist of approximately 302,065 nodes (1,812,390 DoFs) and 278,030 elements.

- ESA's Atmospheric Dynamics Mission Aeolus spacecraft for global wind-component-profile observation, which aims to improve weather forecasting. This spacecraft has a mass of $1800 \mathrm{~kg}$ and the FEM consist of approximately 95,980 nodes (575,880 DoFs) and 109,295 elements.
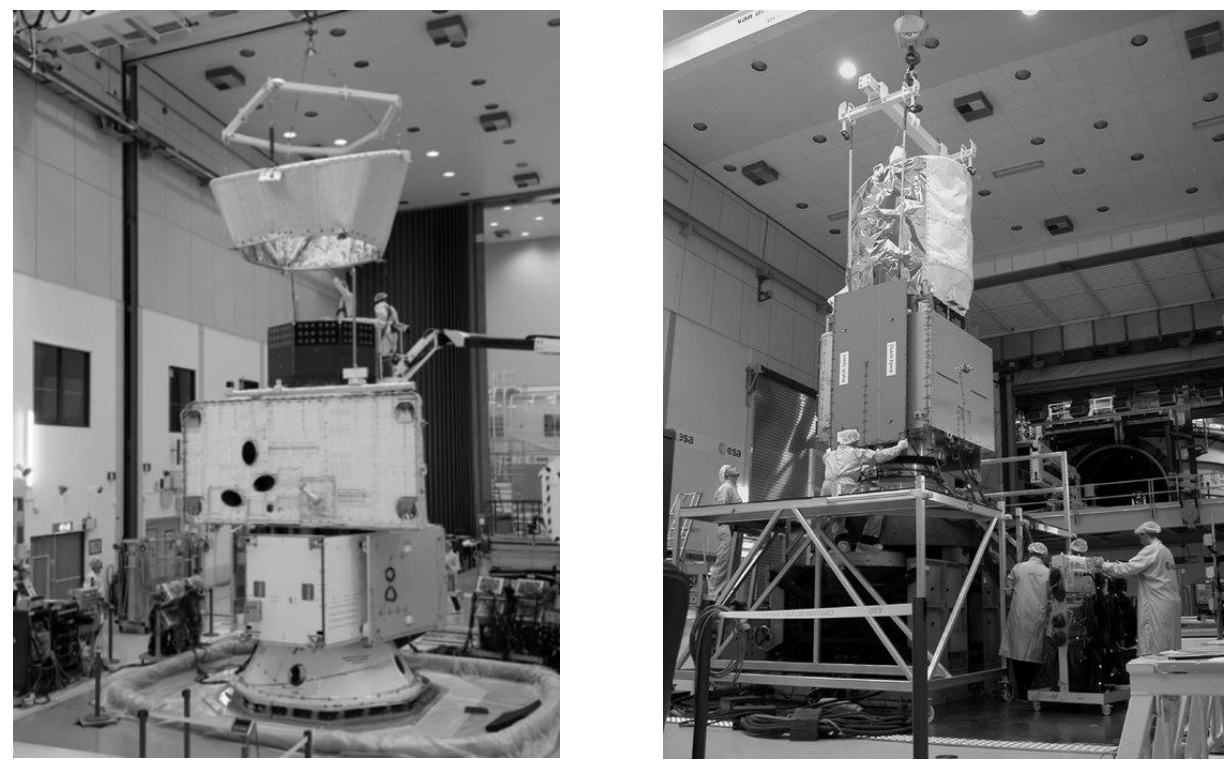

Fig. 1 BepiColombo (left) and Aeolus (right) during vibration test preparation [51] 
These investigations compare the Guyan and SEREP FEM reduction processes in order to assess their suitability for large spacecraft applications. In addition, a number of different sensor location options have also been examined.

Two sets containing the same number of DoFs as the original test MPP have been assessed:

- Both Guyan and SEREP reductions have been performed on the original spacecraft test MPP DoFs. The natural frequencies and mode shapes of the reduced models are compared with the original full FEM results (with mode shapes partitioned to the same MPP DoFs) to assess the accuracy of the reduced model at representing the full system dynamics.

- Alternate sensor locations, with the same number of DoFs as the original MPPs, have been determined through the use of the modal KE approach. With these new sensor locations, the Guyan reduction is again applied to assess whether there is a notable improvement in the orthogonality metric.

Using the initial test MPP DoFs as candidate sets, the effective independence approach is adopted to reduce two new sets half the size of the test MPPs:

- The first 'half MPP sized sets' consists of the DoFs which are selected from the MPPs as being the 'best case' half of the MPP for achieving optimal independence between the target modes.

- The other 'half MPP sized sets' consists of the remaining DoFs, which were omitted from the MPPs during the effective independence sensor selection. As such, this set represents a 'worst case' sensor selection to serve as a comparison with the 'best case' DoFs which were selected from the same original MPPs.

\section{B. Reduction Method Comparisons}

In order to investigate the quality of TAM generated by the Guyan and SEREP reduction processes, modal analyses have been performed on the BepiColombo and Aeolus FEMs. Here, the 'quality assessment' of the reduced models is performed with respect to a consistent full model of each spacecraft. Detailed results are given for BepiColombo, with summaries of results for both spacecraft also presented below. 
Table 1 Modal Effective Masses of Selected Target Modes

\begin{tabular}{|c|c|c|c|c|c|c|c|}
\hline \multicolumn{4}{|c|}{ BepiColombo } & \multicolumn{4}{|c|}{ Aeolus } \\
\hline \multirow{2}{*}{$\begin{array}{c}\text { Freq. } \\
\text { (Hz) }\end{array}$} & \multicolumn{3}{|c|}{ Modal Effective Mass (\%) } & \multirow{2}{*}{$\begin{array}{c}\text { Freq. } \\
\text { (Hz) }\end{array}$} & \multicolumn{3}{|c|}{ Modal Effective Mass (\%) } \\
\hline & TX & TY & $\mathbf{T Z}$ & & TX & TY & TZ \\
\hline 12.68 & 0.66 & 25.96 & 0.02 & 15.96 & 42.35 & 0.02 & 0.00 \\
\hline 13.01 & 25.30 & 0.73 & 0.00 & 16.62 & 0.02 & 45.31 & 0.00 \\
\hline 26.50 & 4.79 & 0.23 & 0.00 & 40.94 & 0.00 & 6.89 & 0.02 \\
\hline 27.12 & 2.40 & 0.55 & 0.00 & 41.30 & 0.00 & 3.40 & 0.02 \\
\hline 27.68 & 1.21 & 0.00 & 0.00 & 48.95 & 1.50 & 0.00 & 0.02 \\
\hline 28.43 & 0.02 & 8.97 & 0.07 & 54.58 & 2.39 & 0.14 & 0.32 \\
\hline 32.43 & 0.11 & 0.10 & 1.21 & 55.63 & 0.75 & 0.15 & 6.76 \\
\hline 33.44 & 0.00 & 0.12 & 5.96 & 56.94 & 3.65 & 0.20 & 0.00 \\
\hline 35.82 & 0.33 & 1.14 & 0.08 & 58.68 & 0.47 & 0.58 & 1.65 \\
\hline 37.03 & 2.91 & 0.01 & 2.94 & 60.00 & 0.00 & 0.00 & 1.84 \\
\hline 37.19 & 0.36 & 0.19 & 3.38 & 61.47 & 0.00 & 0.15 & 1.46 \\
\hline 37.55 & 2.19 & 0.16 & 0.05 & 63.86 & 0.08 & 0.00 & 5.43 \\
\hline 37.77 & 0.50 & 0.52 & 13.15 & 64.35 & 0.47 & 0.00 & 22.90 \\
\hline 38.01 & 0.00 & 1.13 & 1.61 & 64.69 & 5.45 & 0.07 & 2.47 \\
\hline 44.07 & 0.02 & 0.03 & 1.32 & 69.60 & 0.01 & 1.04 & 0.01 \\
\hline 44.37 & 0.16 & 2.34 & 0.01 & 84.64 & 0.26 & 0.05 & 3.65 \\
\hline 45.01 & 0.00 & 0.00 & 1.20 & 85.01 & 0.02 & 0.03 & 1.52 \\
\hline 49.10 & 0.08 & 0.03 & 6.95 & 85.23 & 0.02 & 0.01 & 8.33 \\
\hline 49.77 & 0.00 & 0.12 & 1.03 & - & - & - & - \\
\hline 57.01 & 1.12 & 0.00 & 0.02 & - & - & - & - \\
\hline 57.17 & 2.10 & 0.00 & 0.02 & - & - & - & - \\
\hline
\end{tabular}

For the purposes of this investigation, the modes of interest have been identified based solely on the modal effective mass of each mode in the translational directions. Modes with modal effective mass of at least $1 \%$ in any translational direction have been selected as target modes, as shown above in Table 1 for BepiColombo and Aeolus. The modal effective mass gives an indication to the level of participation of each mode in the loads analysis and is often used to highlight potentially significant modes for correlation [52]. In the case of Spacecraft reduced FEMs delivered for Launcher-Spacecraft CLA's, adequate effective mass capture is deemed 'mandatory' for adequate representation of coupled behavior.

Initially, the starting set of DoFs are those which correspond to the original test MPP of accelerometer positions used during the spacecraft sine-sweep tests. With Guyan reduction, the number of reduced modes identified within the frequency range of interest is dependent on which DoFs are being retained in the reduction; hence MAC assessments have been performed to determine which reduced mode matched most closely the corresponding selected full target mode (partitioned to the MPP DoFs). It should be noted that when using MAC to determine the 'best match' Guyan reduced mode for each target full (partitioned) mode, it was found that in some cases the same reduced mode gave the highest MAC for more than one of the target modes. As a result, some reduced modes have been repeated in order that the best match is given for each target mode in turn. For SEREP reduction, only the 
selected target modes have been included in the modal matrix used to derive the reduced modes and thus only the required reduced modes are present and no mode matching is required.

Comparisons have been made between the natural frequencies of the full (partitioned) target modes and the reduced modes selected as 'best match' to these targets based on MAC comparisons. Fig. 2 illustrates the reduced model natural frequencies plotted against those calculated from the full FEM, for both BepiColombo (left) and Aeolus (right).

The MAC for the matched reduced modes compared with the full partitioned modes of BepiColombo is given in Fig. 3 for both Guyan and SEREP reductions. The leading diagonal values provide an indication of how well the 'matched' reduced modes represent their targets partitioned from the full FEM results. Fig. 4 shows the orthogonality check results obtained using the Guyan and SEREP reduced TAMs. The reduced and full modes are again compared, but now with the reduced mass matrix providing a relative weighting to the DoFs and producing an orthogonality check, in which the off-diagonal values are important indicators of the TAM quality. The full modes are then compared with themselves, using both TAMs to give an indication of the TAM quality.

\section{BepiColombo}

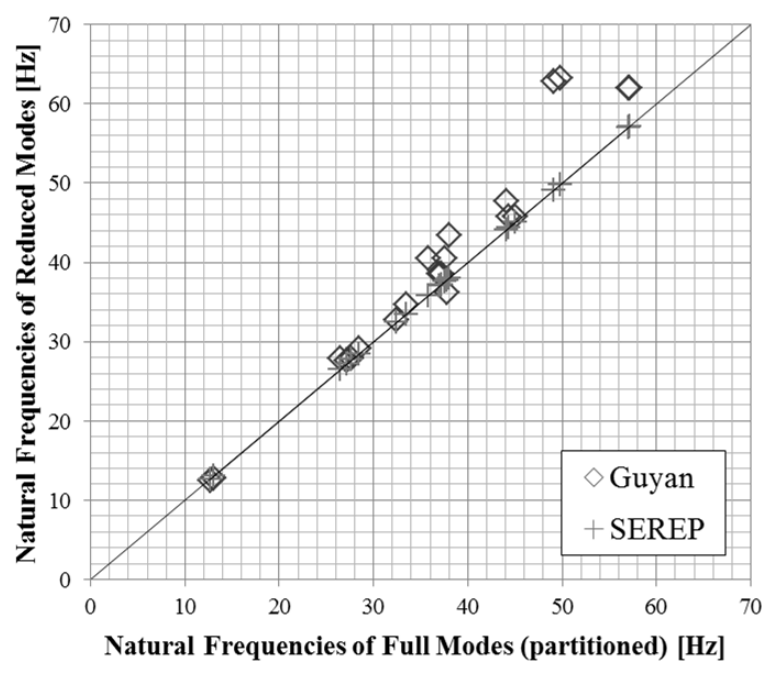

Aeolus

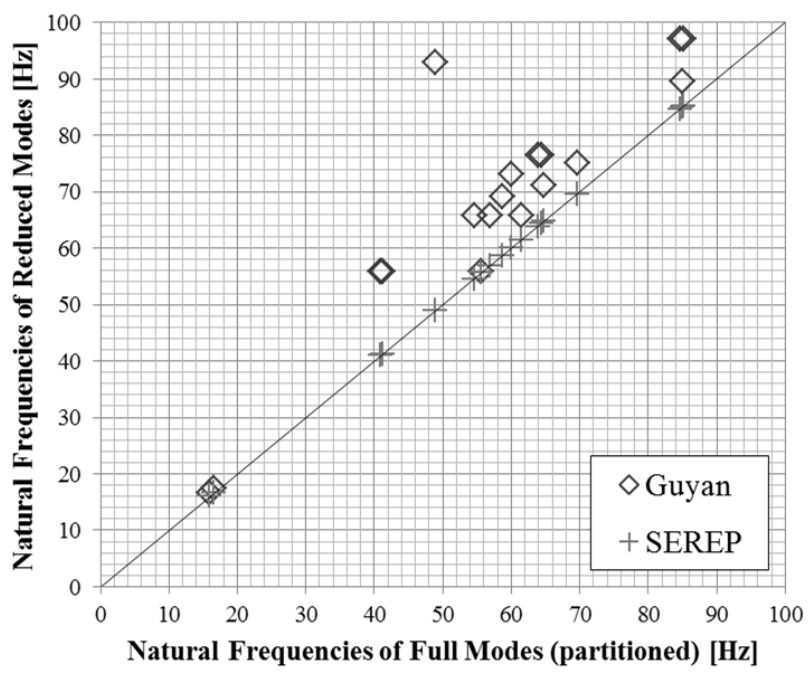

Fig. 2 Natural Frequencies (Hz) for Reduced vs Full FEM

From Fig. 2 it can be noted that for the SEREP natural frequencies the points all lie on the diagonal, indicating a perfect match. For the Guyan reduction, some points deviate from the diagonal indicating a difference between the reduced and full model results. The natural frequencies of the Guyan reduced models match the targets increasingly poorly as the frequency is increased; for example going from an almost exact match for the first target mode of BepiColombo at $12.7 \mathrm{~Hz}$, to a maximum difference of $28 \%$ from the target at $49.8 \mathrm{~Hz}$, with a similar trend found for 
Aeolus. In general, the static nature of the Guyan reduction, with the neglected inertia of omitted DoFs would be expected to lead to inaccuracies and over prediction of natural frequencies as frequencies grow higher, but these results serve to highlight the issue in the frequency range of interest.
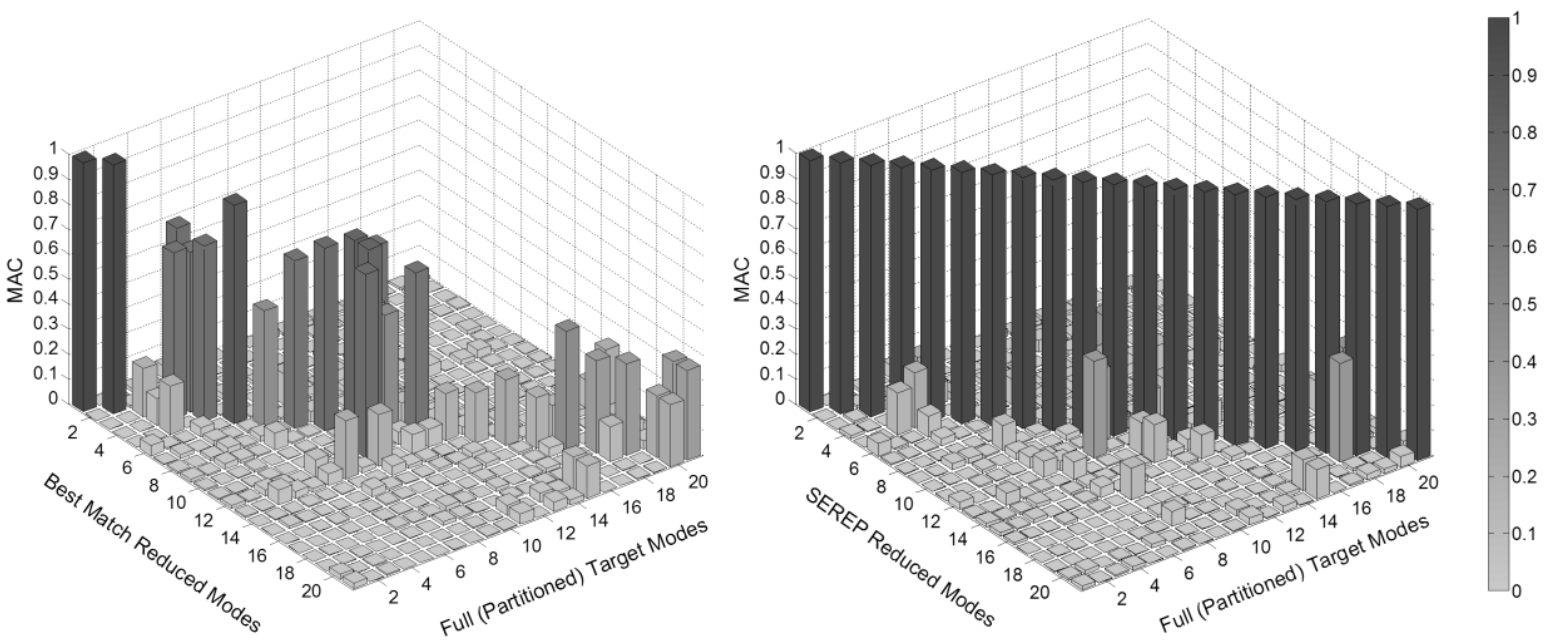

Fig. 3 MAC of reduced and full FEM mode shapes for BepiColombo. Left: Guyan, Right: SEREP. 

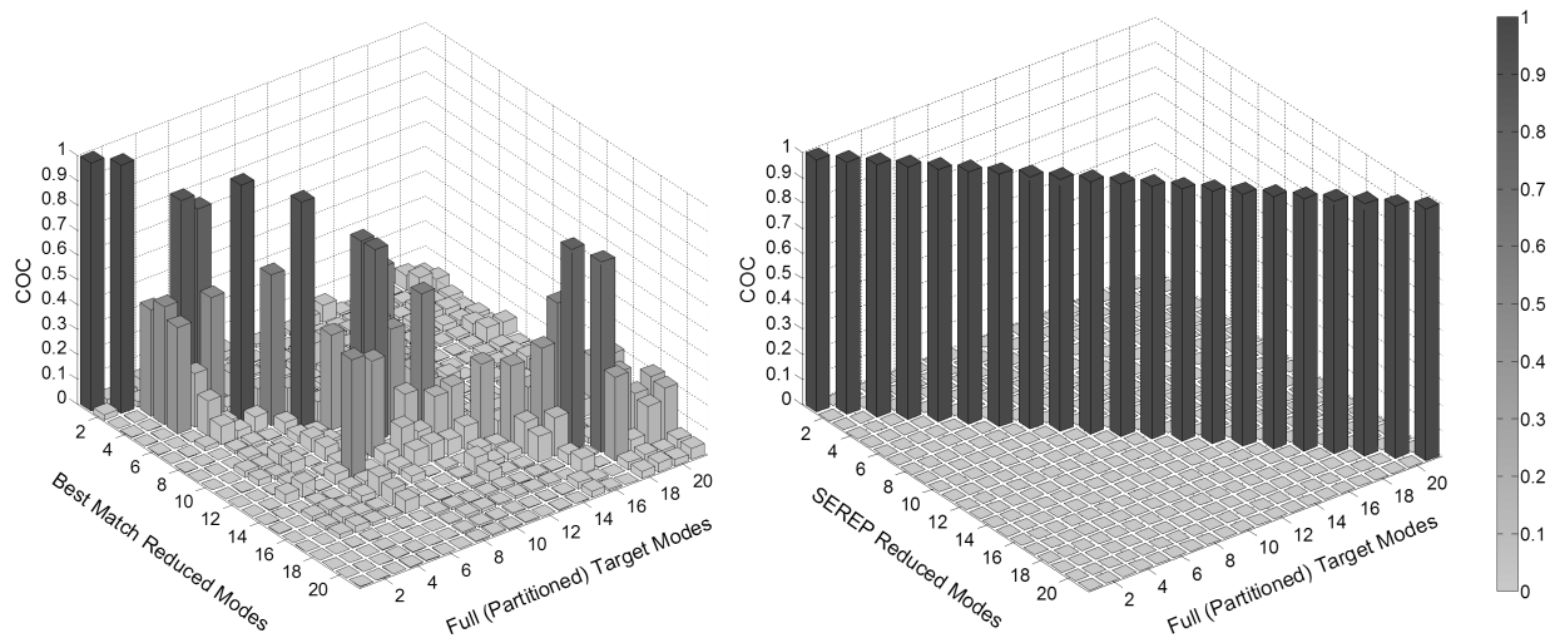

(a) Partition of Full Modes vs Reduced Modes. Left: Guyan, Right: SEREP
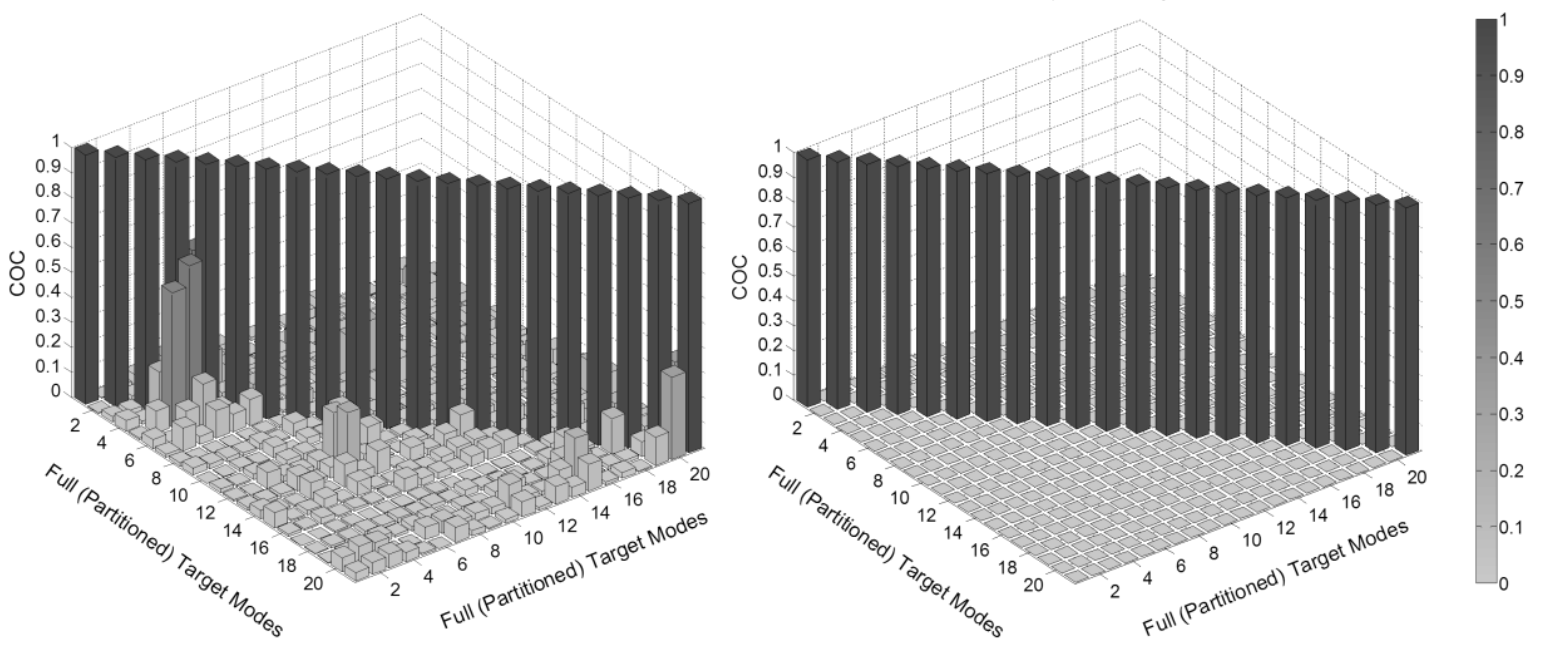

(b) Partition of Full Modes vs Partition of Full Modes. Left: Guyan, Right: SEREP

Fig. 4 Cross-orthogonality results for BepiColombo using reduced TAMs. Left: Guyan, Right: SEREP.

The MAC (Fig. 3) and COC (Fig. 4 (a)) comparisons of the partitioned full and Guyan reduced modes revealed a poor match in modes other than the first few fundamental modes. This is a notable finding as it demonstrates the errors introduced as a result of the reduction process, which means that the reduced model is not representative of the full model. Thus, any comparisons between the reduced FEM and test modes are not necessarily indicative of how representative of the real spacecraft the full FEM actually is, even with as many as approximately 400 and 300 test instrumentation DoFs for BepiColombo and Aeolus respectively.

In contrast, the SEREP method has also been implemented to reduce the FEM to the test measured DoFs. Here the reduced model matches exactly the full model results for natural frequencies and mode shapes. This is an 
inherent aspect of the SEREP process and means that the reduced model is representative of the full model, making comparisons between reduced model and test results potentially more meaningful.

\section{Kinetic Energy Sensor Placement Influence on FEM Reductions}

Although the above results should to some extent be expected, the findings serve to highlight the extent of the lack of accuracy in the Guyan representation of the spacecraft dynamics when the model is reduced to the DoFs used in the original spacecraft sine sweep test MPP as used in practice. In order to determine whether these results are indicative of inherent issues in using Guyan reduction for large spacecraft applications, or merely a consequence of poorly selected sensor locations in the original MPP, not well suited to this reduction type; the use of a different sensor location set, selected on the basis of modal $\mathrm{KE}$, has been investigated. In order to ensure comparisons are meaningful, the number of DoFs in this new KE based sensor set matches that of the original MPP. For BepiColombo, this means approximately 400 DoFs selected based on kinetic energy, and for Aeolus a set of approximately 300 DoFs.

AutoMAC is the comparison of the full FEM mode shapes, partitioned to the sensor set DoFs, with themselves through use of the MAC correlation assessment criteria [1]:

$$
\text { AutoMAC }=\frac{\left(\phi_{a}{ }^{\mathrm{T}} \boldsymbol{\phi}_{a}\right)^{2}}{\left(\boldsymbol{\phi}_{a}{ }^{\mathrm{T}} \boldsymbol{\phi}_{a}\right) \cdot\left(\boldsymbol{\phi}_{a}{ }^{\mathrm{T}} \boldsymbol{\phi}_{a}\right)}
$$

The leading diagonal will, inherently, yield all unity as each mode perfectly matches itself; it is therefore the offdiagonal terms which are of interest. High off-diagonals indicate a lack of independence between the mode shapes and, therefore, imply a potentially poor choice of sensor locations. As such, low off-diagonals reveal a good level of independence between the modes for the selected MPP.

Fig. 5 below shows 3D bar plots of AutoMAC comparisons for the BepiColombo spacecraft target modes (as identified in Table 1). The first image, on the left, shows the AutoMAC for the original MPP while the second image, on the right, is the result of the same number of DoFs but instead selected using the KE method. It can be seen that there are some higher off-diagonal values in the right hand image compare to those on the left plot. The same deterioration was found for the Aeolus spacecraft when the KE rather than MPP DoF set was applied, as summarized in Table 2. This indicates that the KE method has resulted in a sensor set which makes distinguishing the different modes more difficult than it was for the original MPP. This is to be expected, as the KE method does not optimize based on linear independence between modes, but purely on mode signal strength. 

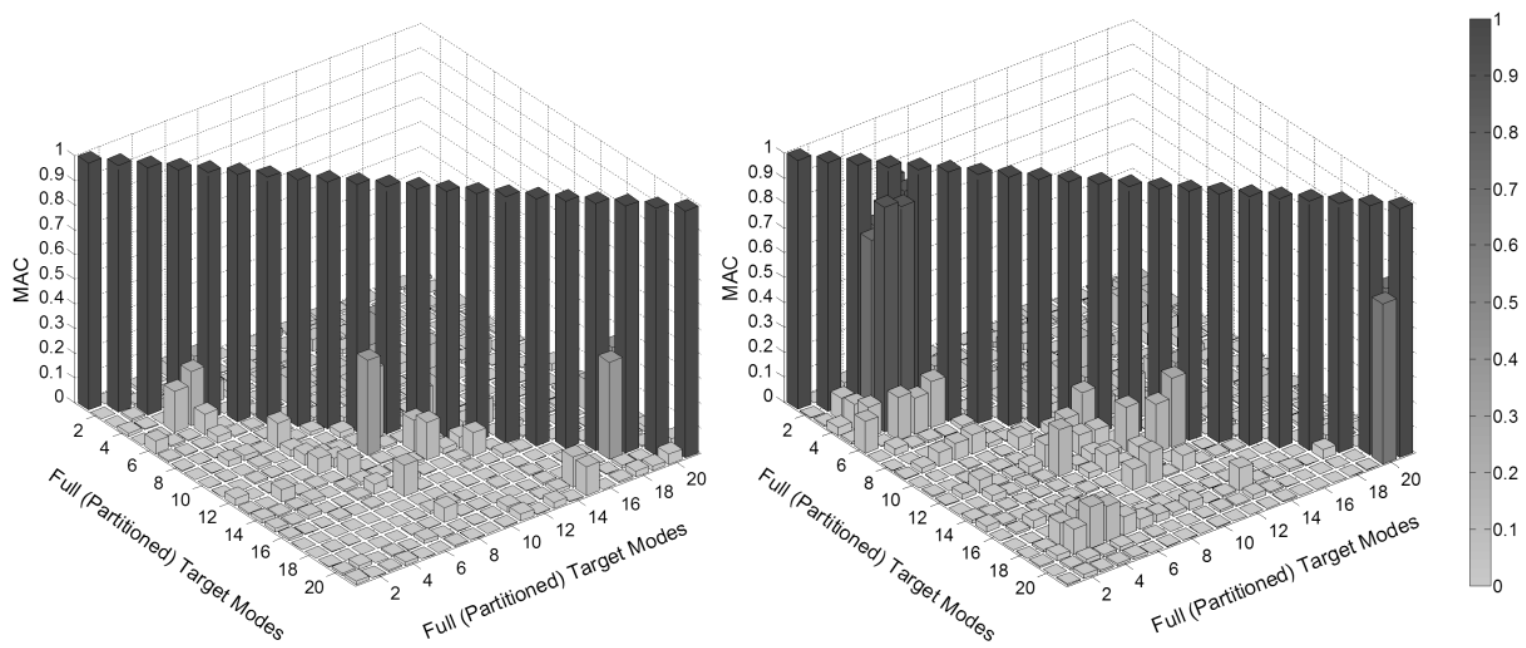

Fig. 5 AutoMAC for BepiColombo target modes. Left: MPP DoFs. Right: KE DoF Selection.

The MAC comparisons for BepiColombo of the full model target mode shapes, partitioned to these new KE DoF sets, with the best matched Guyan reduced modes are given in Fig. 6. The original MPP MAC plot is also included again for comparison. The corresponding COCs, performed using the respective Guyan TAMs, are given in Fig. 7. For added clarity, these results are also summarized in Table 2.
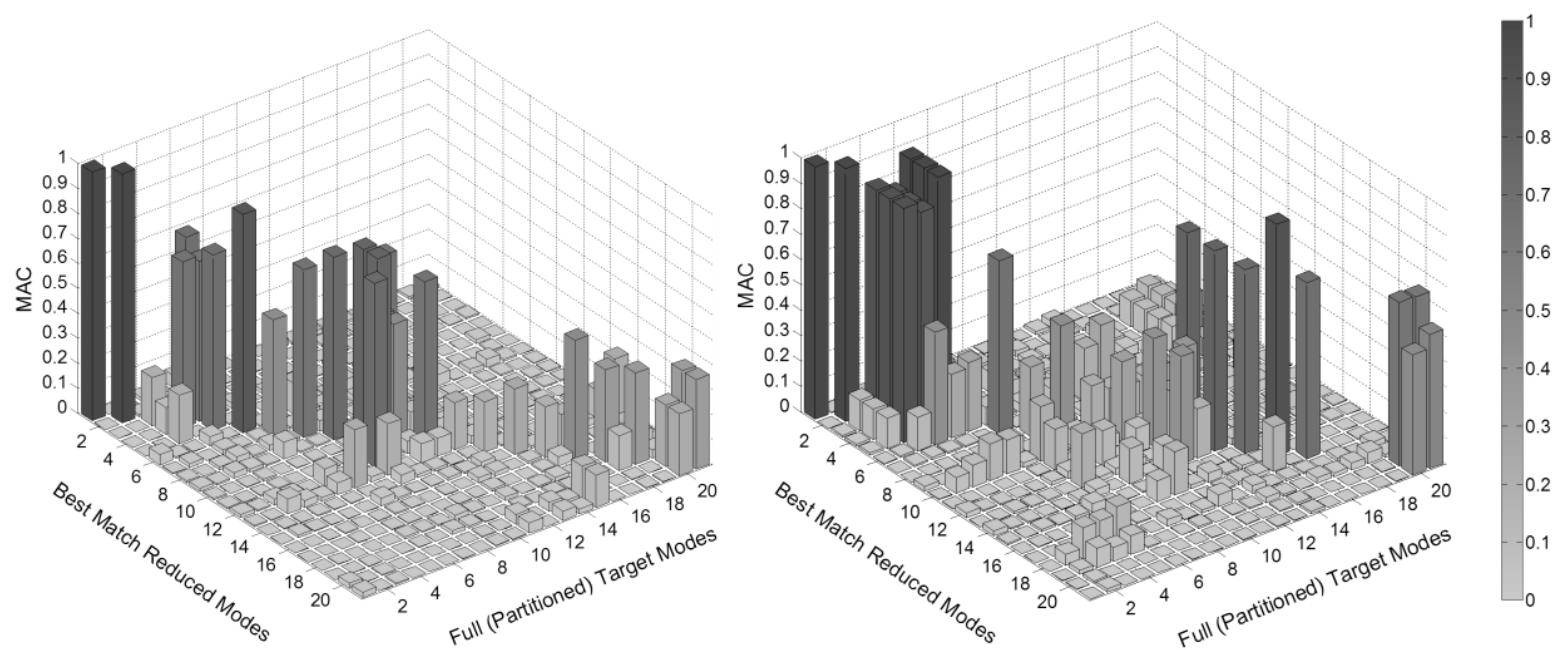

Fig. 6 MAC for BepiColombo Full vs Guyan reduced target modes. Left: MPP, Right: KE Selected. 

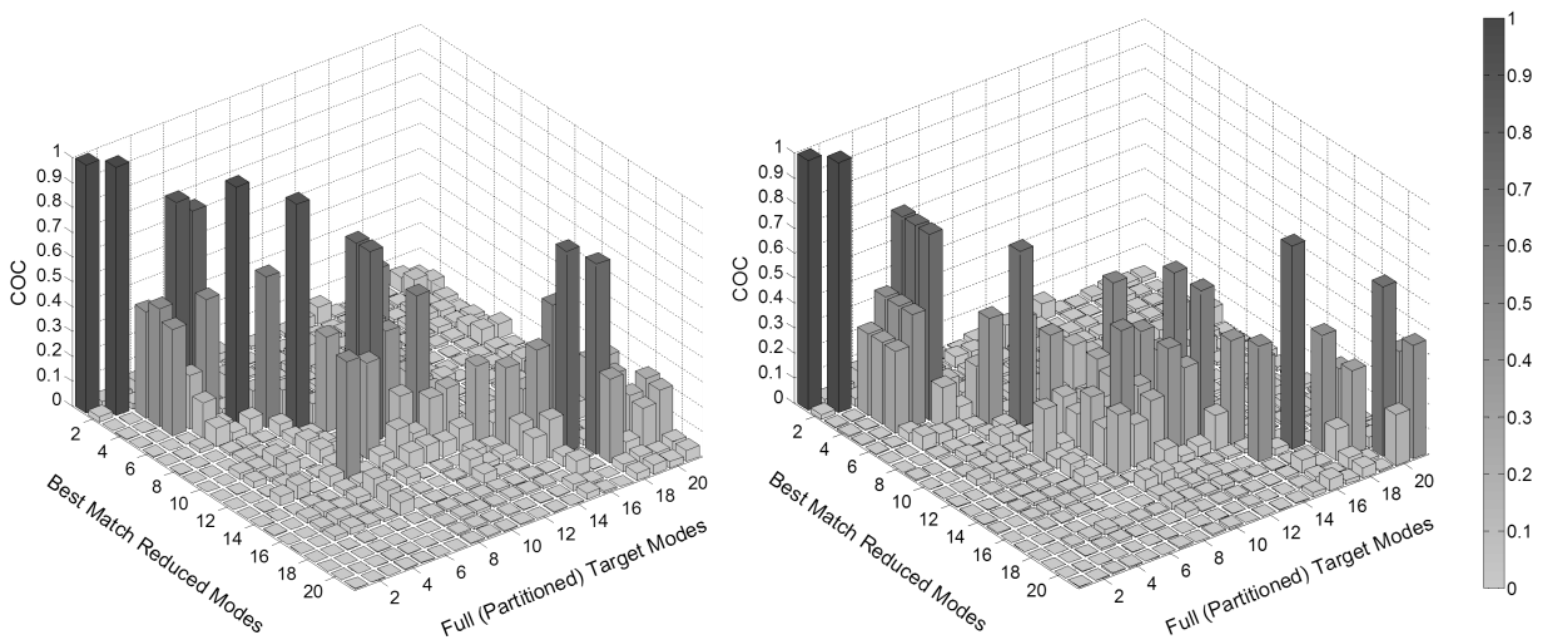

(a) Partition of Full Modes vs Guyan Reduced Modes. Left: MPP, Right: KE Selected
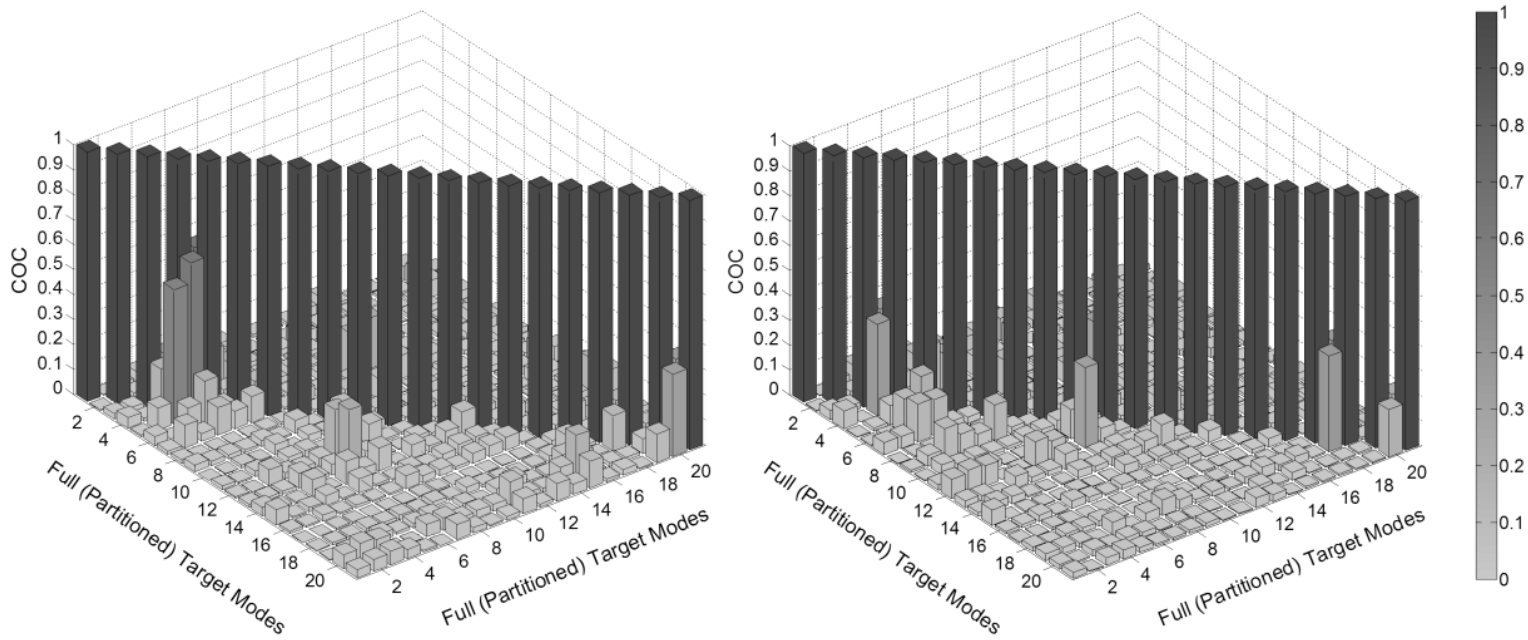

(b) Partition of Full Modes vs Partition of Full Modes. Left: MPP, Right: KE Selected

Fig. 7 Orthogonality checks for BepiColombo using Guyan TAM. Left: MPP, Right: KE Selected. 
Table 2 Summary of results for KE selected reduced sensor sets for both spacecraft

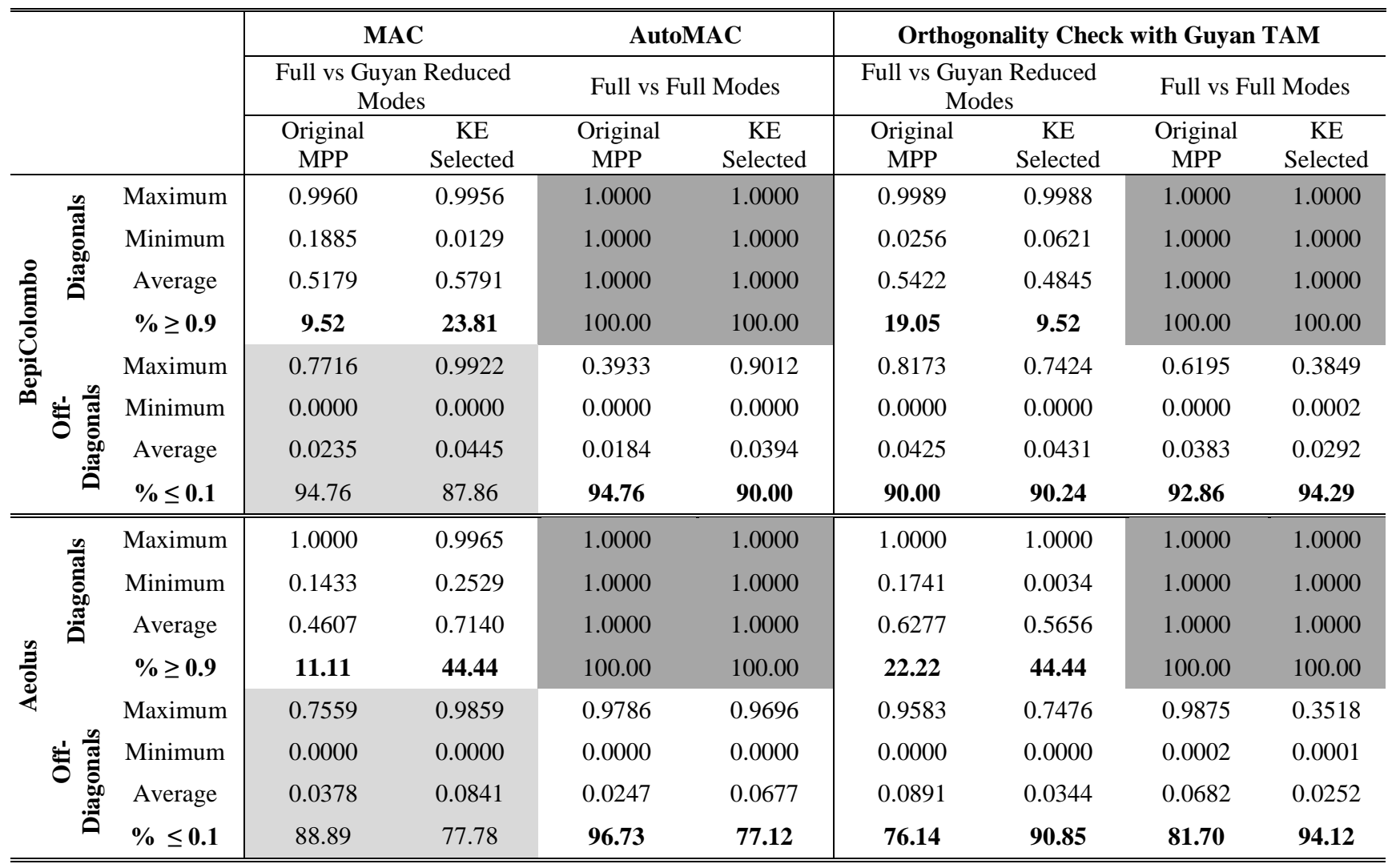

The use of modal kinetic energy to determine a new sensor set placement has not resulted in a marked improvement in the accuracy of the Guyan TAM at representing the full FEM. For BepiColombo, the summary in Table 2 reveals no notable improvement in orthogonality check results. There is a slight improvement in the MAC between reduced and corresponding target modes, however this is at the cost of less independence between target modes. For Aeolus, the potential benefits of the modal KE approach to sensor placement are more pronounced; with general increases in diagonal terms and decreases in average off-diagonal COC values. Overall, however, it is still observed that, even with this optimized reduced DoF selection, the Guyan reduction does not achieve an accurate representation of the full system for more than a few of the target modes of either spacecraft.

It should be noted that these comparisons have focused on Guyan reduction and SEREP has not been included. This is the case as SEREP produces reduced analytical mode shapes and reduced TAM which inherently achieve perfect orthogonality with respect to the full FE model modes regardless of sensor selection. Any SEREP results would only serve to confirm the results Fig. 3 and Fig. 4, and as such are omitted here for brevity. 


\section{Effective Independence Sensor Placement Influence on FEM Reductions}

The sensor set locations can have an influence of the quality of TAM generated through model reduction. The influence of sensor set placement for optimum linear independence between modes has also been investigated through the use of the two newly generated sensor sets per spacecraft. For BepiColombo these sets comprise: the 'best case' approximately 200 DoFs chosen in an EfI method selection based on a candidate set of the original approximately 400 DoFs in the BepiColombo MPP; and an alternative set with the remaining approximately 200 DoFs which were eliminated in the EfI selection. Likewise for Aeolus, the same approach has been applied to obtain two subsets of approximately 150 DoFs each from the original approximately 300 DoF MPP. In order to assess the linear independence of target modes, which the EfI method aims to optimize, AutoMAC checks are performed.
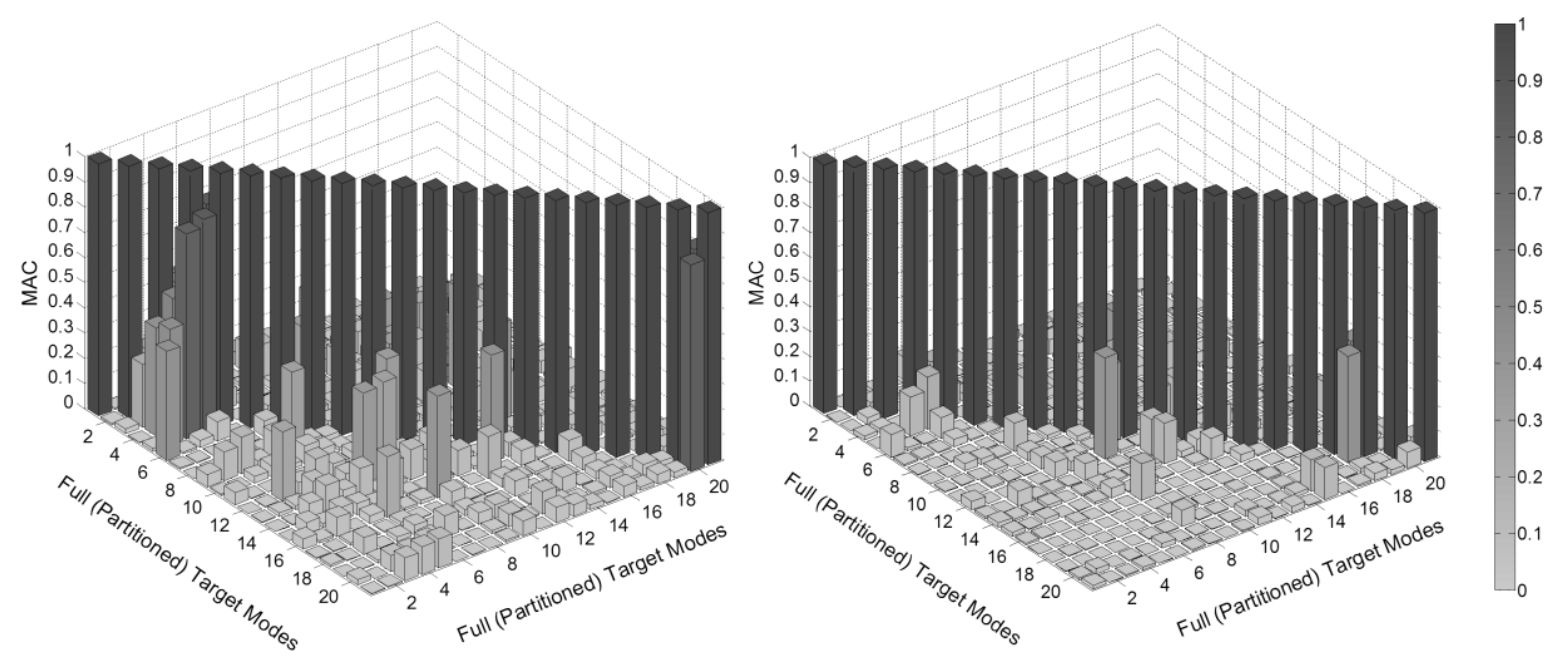

Fig. 8 AutoMAC for BepiColombo target modes. Left: EfI Eliminated. Right: EfI Selection.

Fig. 8 above shows 3D bar plots of AutoMAC comparisons for the BepiColombo spacecraft target modes (as identified in Table 1). The original MPP had approximately 400 accelerometers and, therefore, the original FEM was reduced to approximately 400 DoFs. Here this has been reduced further to include only approximately 200 DoFs, selected from the candidate set of original approximately 400 DoFs in the MPP. The first image, on the left, shows a selection derived from the DoFs eliminated by the EfI method while the second image, on the right, is the result of the other DoFs selected using the EfI method. It can be seen that although there are still some noticeable off-diagonal values in the right hand image, these are significantly reduced from those on the left plot. The left plot in Fig. 8 shows that the EfI selected DoF set result in a plot closely resembling the AutoMAC for the full MPP, as given previously in Fig. 5, further highlighting the lack of independent information contained in the EfI omitted DoF 
set. The same improvement was found for the Aeolus spacecraft, as summarized in Table 4. This comparison can be useful to check the independence of modes resulting from proposed MPPs, and is therefore a valuable aid in comparing different sensor set location options.

When the Guyan reduction process is applied for the new smaller sensor subsets, even fewer distinct modes are identified by the finite element modal analysis in the frequency range of interest than for the original MPP, as shown in Table 3. It should also be noted that, for both spacecraft, the FEA was able to identify significantly more reduced modes for the EfI selected DoF set, than that of the DoFs eliminated by the EfI method.

Table 3 Number of modes identified in Guyan reduced model in FEA frequency range of interest

\begin{tabular}{cccccc}
\hline \hline & & \multicolumn{2}{c}{ BepiColombo } & Aeolus \\
\hline $\begin{array}{c}\text { Type of } \\
\text { Run }\end{array}$ & DOFs Used & $\begin{array}{c}\text { Number of DOFs } \\
\text { (approx.) }\end{array}$ & $\begin{array}{c}\text { Number of Modes } \\
\text { Identified by FEA } \\
(0-100 H z)\end{array}$ & $\begin{array}{c}\text { Number of DOFs } \\
\text { (approx.) }\end{array}$ & $\begin{array}{c}\text { Number of Modes } \\
\text { Identified by FEA } \\
(0-100 \mathrm{~Hz})\end{array}$ \\
\hline Full Run & Full Model & 100s of thousands & 247 & 100 s of thousands & 197 \\
\hline \multirow{4}{*}{$\begin{array}{c}\text { Guyan } \\
\text { Reduction }\end{array}$} & KE from Full Model & 400 & 105 & 300 & 50 \\
& EfI Selected from MPP & 200 & 125 & 300 & 88 \\
& EfI Omitted from MPP & 200 & 93 & 150 & 46 \\
\hline \hline
\end{tabular}
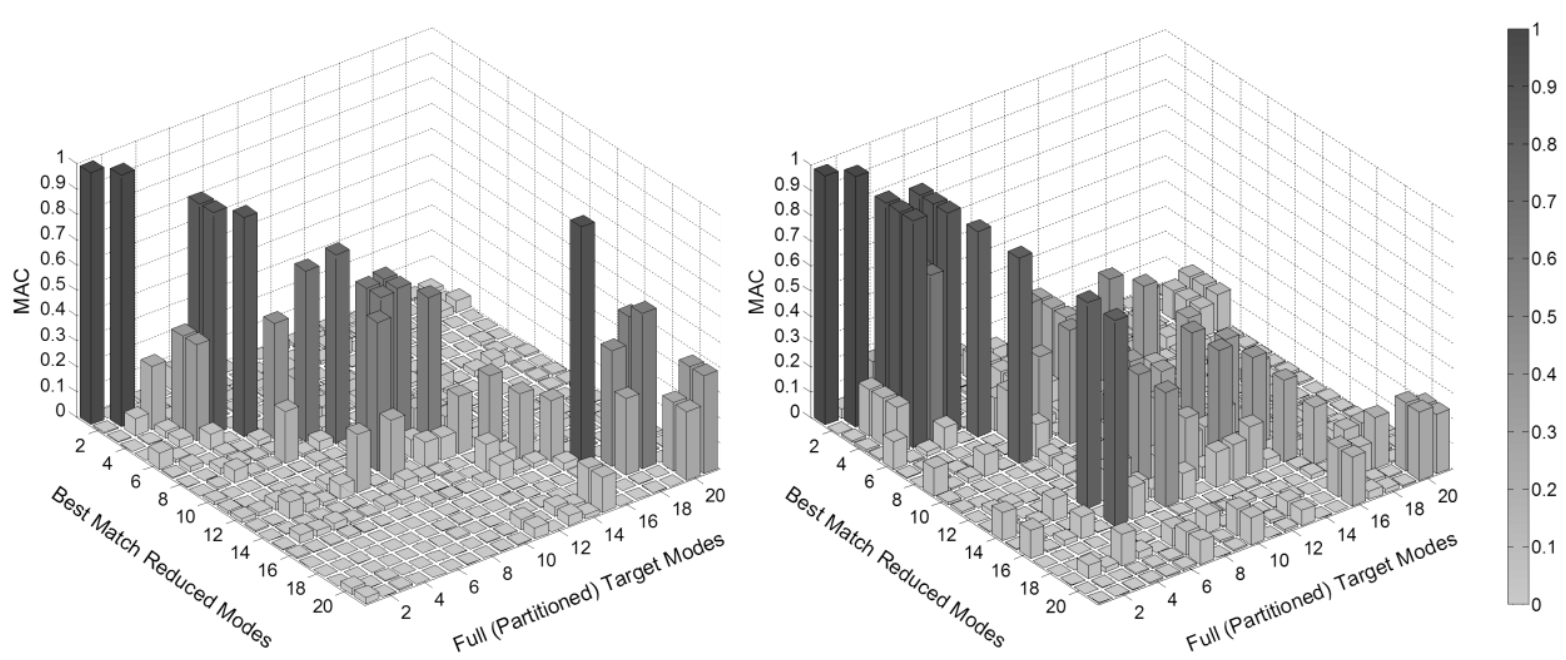

Fig. 9 MAC for BepiColombo Full vs Guyan reduced modes. Left: EfI Selected, Right: EfI Eliminated.

The MAC comparisons for BepiColombo of the full model target mode shapes, partitioned to these new smaller DoF sets, with the best matched Guyan reduced modes are given in Fig. 9. The corresponding COCs, performed using the respective Guyan TAMs, are given in Fig. 10. For added clarity, these results are also summarized in Table 4. 
The below results show that, even within the initial BepiColombo MPP sensor set, there is a notable difference in the quality of reduced Guyan model depending on the selected subset of sensors. For both spacecraft, a higher percentage of the leading diagonal terms are over 0.9 for the EfI selected sensor set, and the off-diagonals are lower, both for AutoMAC and orthogonality checks compared to the alternative sensor set.
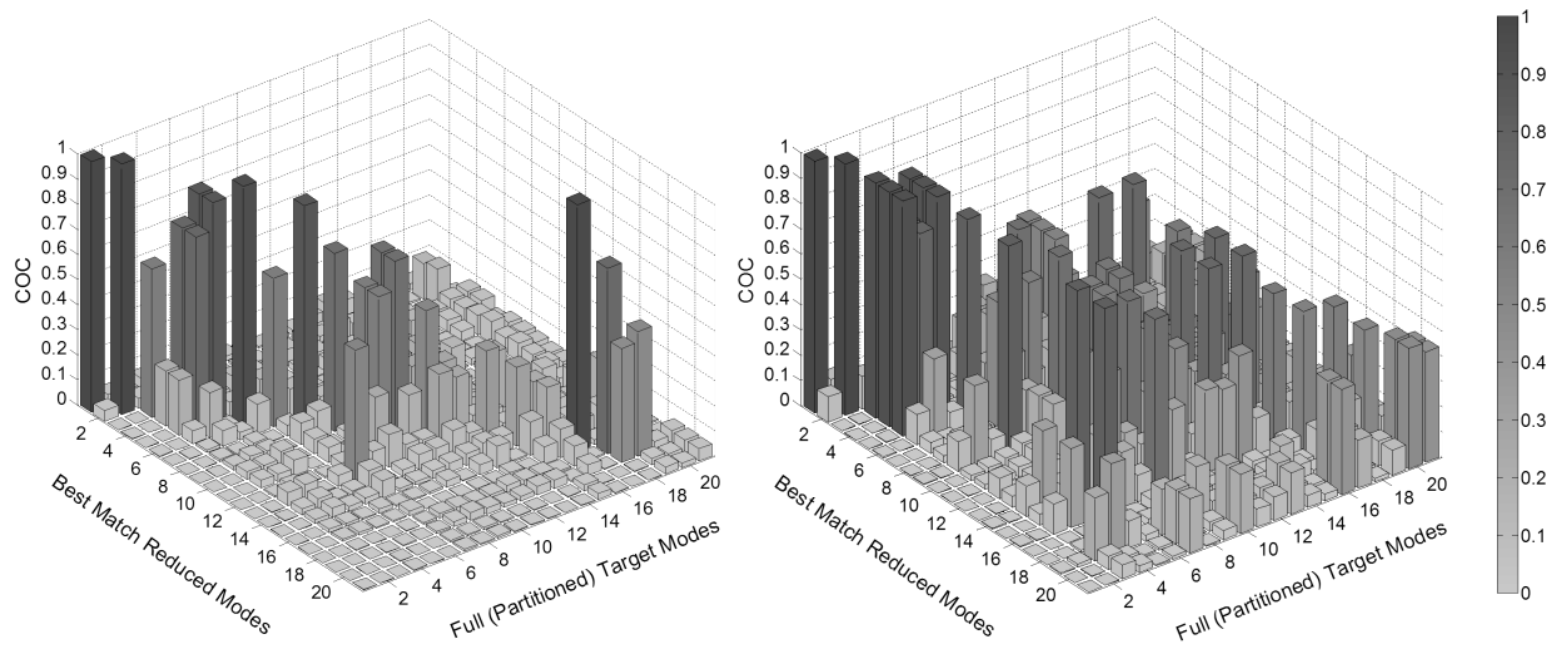

(a) Partition of Full Modes vs Guyan Reduced Modes. Left: EfI Selected, Right: EfI Eliminated
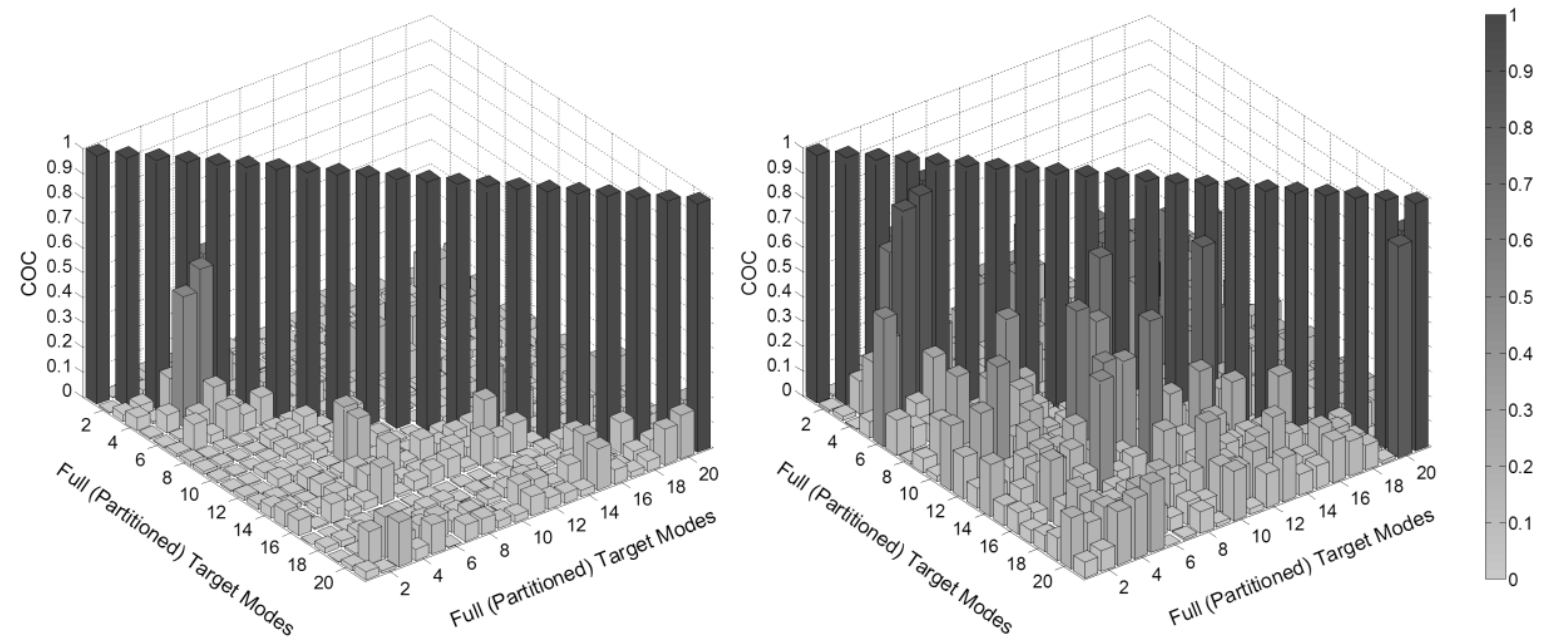

(b) Partition of Full Modes vs Partition of Full Modes. Left: EfI Selected, Right: EfI Eliminated

Fig. 10. Orthogonality checks for BepiColombo using Guyan TAM. Left: EfI Selected, Right: EfI Eliminated.

Again these comparisons have focused only on the quality of Guyan reduced TAMs as SEREP produces reduced mode shapes and reduced TAMs which inherently achieve perfect match and are orthogonal to the partitioned full mode vectors for any reduced DoF set. 
Table 4 Summary of results for EfI selected reduced sensor sets for both spacecraft

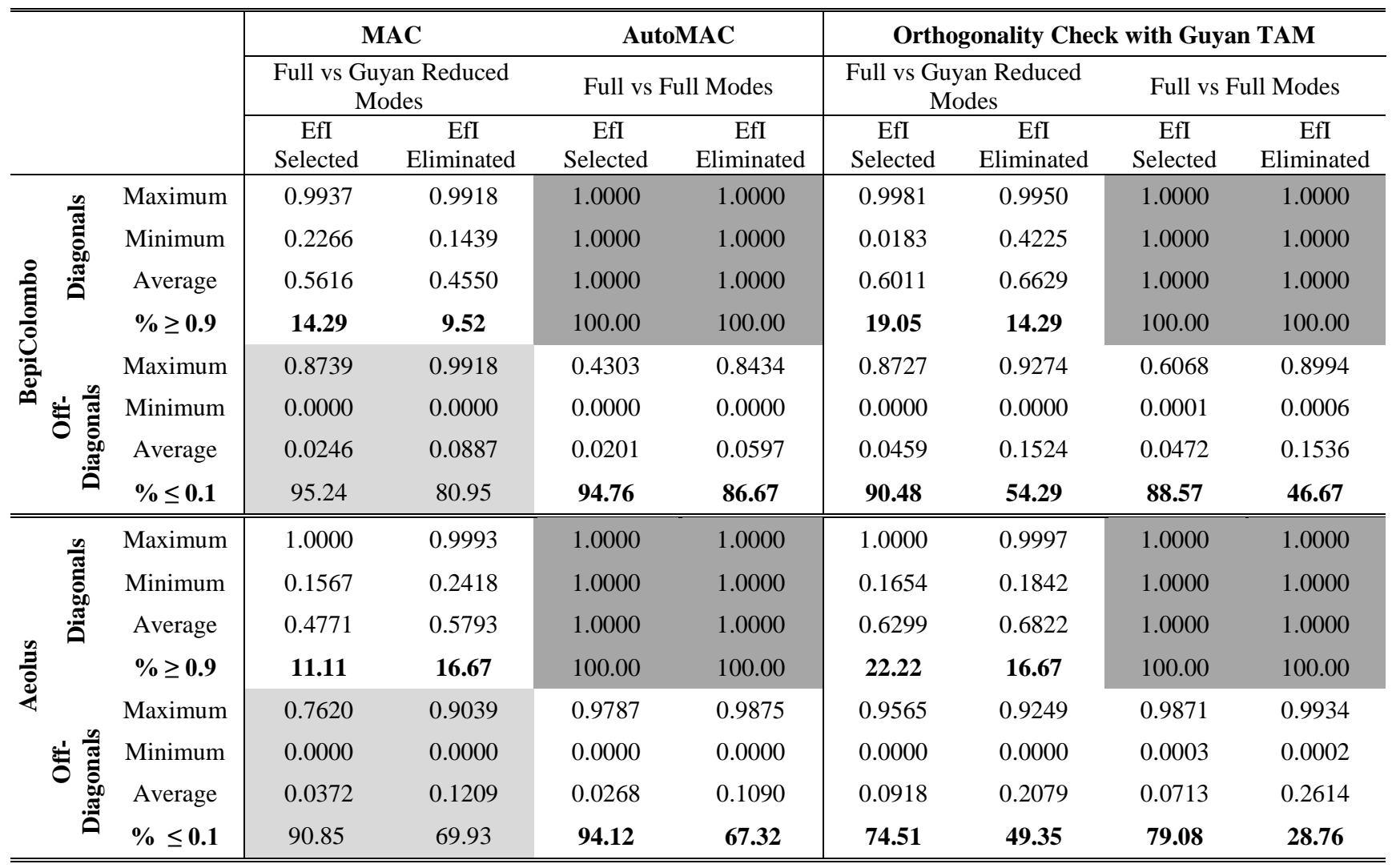

\section{E. Orthogonality Check Comparisons of Test and FEM using both Guyan and SEREP Reduction Methods}

All of the previous results presented herein have demonstrated the ability of the reduced TAM to represent the full FEM for the purpose of COCs solely through FEM-only comparisons. The same Guyan and SEREP reduced TAMs used in those investigations have also been used to compare the FEM mode shapes (from analyses of the full models, with the modal vectors partitioned to the required DoFs matching the test measured locations) with the mode shapes extracted from the test measured data.

The experimental results used in these comparisons are derived from data captured from MPP accelerometers used during base-shake sine sweep testing of the considered spacecraft. In order to obtain modal information from the resulting FRFs, appropriate curve-fitting methods were employed. In this case, the focus is on comparison with FEM normal modes, as such the normalized test modes are extracted. The modal parameter estimation was of particular importance given the high modal density, particularly with BepiColombo, requiring the implementation of MDOF curve-fitting techniques [53]. As such, the extraction of mode shapes used in this study has been achieved mainly through the use of the poly-reference least squares complex frequency method [54], which is a popular 
approach for structures such as those considered herein. Despite the use of one of the most popular current methods, the modal extraction provides only a 'best-fit' estimation of the test modes from the available data [50].

As for the purely analytical comparisons presented in the previous section, again the COCs have been conducted for:

- The full MPP sets; containing all of the DoFs at which accelerometers captured the response of the structural thermal models.

- The EfI selected half sets; which resulted from applying the EfI method to the MPPs in order to identify the 'best half' DoFs from the MPP.

- The EfI Eliminated half sets; which were those remaining 'worst half' DoFs which were not selected when the EfI method was applied to the MPPs.

The modes of interest have again been selected on the basis of modal effective mass, with FEM modes achieving $>5 \% \mathrm{~m}_{\text {eff }}$ in any translational direction (see Table 1) and the best matching corresponding test modes being included in the test-FEM comparison. The results are summarized in Table 5.

From Table 5 it should be noted that, despite only containing half of the DoFs, the EfI Selected DoF set achieves very similar results to the Full MPP set. This is most clearly evident for Aeolus, but is also demonstrated in the average, maximum and minimum diagonal values for BepiColombo.

Both the Full MPP and EfI Selected sets show the SEREP TAM achieves markedly higher leading diagonal values than the orthogonality checks conducted with Guyan TAM but at the cost of the off-diagonal values also being slightly higher for SEREP than Guyan. For the EfI Eliminated sets these trends are reversed, with higher cross-orthogonality values in Guyan (both diagonal and off-diagonal) and lower diagonal values for SEREP. It should, however, be noted that the Guyan reduced TAMs used to generate the EfI Eliminated results are those demonstrated previously (see Table 4) as giving the poorest representation of the full FEM. 
Table 5 Summary of results comparing test and finite element analyses for both spacecraft

\begin{tabular}{|c|c|c|c|c|c|c|c|c|}
\hline & & & \multicolumn{6}{|c|}{ Full FEM Modes (partitioned to required degrees of freedom) vs Test Modes } \\
\hline & & & \multicolumn{3}{|c|}{ Orthogonality Check with SEREP TAM } & \multicolumn{3}{|c|}{ Orthogonality Check with Guyan TAM } \\
\hline & & & Full MPP & EfI Selected & EfI Eliminated & Full MPP & EfI Selected & EfI Eliminated \\
\hline \multirow{8}{*}{ فํ. } & \multirow{3}{*}{ 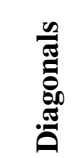 } & Maximum & 0.9821 & 0.9811 & 0.9115 & 0.9123 & 0.9639 & 0.9817 \\
\hline & & Minimum & 0.4396 & 0.4047 & 0.0084 & 0.0333 & 0.0231 & 0.0510 \\
\hline & & Average & 0.8000 & 0.7769 & 0.4854 & 0.6132 & 0.6094 & 0.6883 \\
\hline & \multirow{5}{*}{ 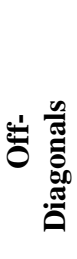 } & $\% \geq 0.9$ & 50.00 & 50.00 & 16.67 & 33.33 & 16.67 & 33.33 \\
\hline & & Maximum & 0.1962 & 0.2353 & 0.1426 & 0.1882 & 0.2147 & 0.4365 \\
\hline & & Minimum & 0.0002 & 0.0027 & 0.0015 & 0.0010 & 0.0026 & 0.0009 \\
\hline & & Average & 0.0757 & 0.0947 & 0.0466 & 0.0405 & 0.0585 & 0.1139 \\
\hline & & $\% \leq 0.1$ & 73.33 & 53.33 & 93.33 & 90.00 & 83.33 & 60.00 \\
\hline \multirow{8}{*}{$\frac{n}{3}$} & \multirow{4}{*}{ 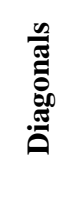 } & Maximum & 0.9918 & 0.9911 & 0.6386 & 0.9687 & 0.9724 & 0.9906 \\
\hline & & Minimum & 0.3283 & 0.3549 & 0.0163 & 0.0677 & 0.0241 & 0.0842 \\
\hline & & Average & 0.6561 & 0.6583 & 0.3341 & 0.5677 & 0.5608 & 0.6424 \\
\hline & & $\% \geq 0.9$ & 37.50 & 37.50 & 0.00 & 25.00 & 25.00 & 37.50 \\
\hline & \multirow{4}{*}{ 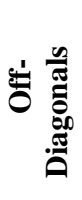 } & Maximum & 0.7385 & 0.7315 & 0.5503 & 0.6767 & 0.6609 & 0.9033 \\
\hline & & Minimum & 0.0029 & 0.0006 & 0.0018 & 0.0002 & 0.0016 & 0.0124 \\
\hline & & Average & 0.1256 & 0.1287 & 0.1643 & 0.0931 & 0.0917 & 0.2484 \\
\hline & & $\% \leq 0.1$ & 58.93 & 60.71 & 48.21 & 75.00 & 73.21 & 37.50 \\
\hline
\end{tabular}

It should be re-iterated that, in practice, the ultimate purpose of these COCs is the correlation and update of the FEM for use in subsequent analyses to ensure the structure is able to withstand events which cannot be fully replicated in the test environment. As such, it is vital that the reduced models are giving a true representation of the full FEM in order that genuine differences between test and FEM are highlighted for further investigation, as well as being robust enough not to be adversely influenced by inevitable minor inconsistencies in test and FEM responses.

During correlation activities it was found that many of the required FEM updates, identified through examination of many correlation criteria including COCs such as those presented herein, related to issues with initial modelling assumptions [55]. One of the more common issues in both spacecraft FEMs is the internal boundary conditions; particularly in relation to internal joints represented through the use of rigid body RBE2 elements. The common application of 'RBE2 spiders' to provide connections was found, in certain cases, to result in the introduction of 'point flexibilities' at the connecting nodes/GRID points of the FEM structure. In BepiColombo, for example, the result was that the eigen-frequencies of some regions of the structure were lower than was seen in the test. Although in itself this could be considered a relatively minor issue, the frequency shift resulted in the local modes is different 
regions of the stacked structure coupling in a manner which was not representative of what was seen in the test. Additionally, the assumptions surrounding the addition of non-structural mass also required FEM update in order to address similar unrepresentative coupling of local modes. In the case of BepiColombo the manner of using 'smeared' non-structural mass to represent the multi-layer insulation on a panel resulted in unrepresentative coupling with the vibration modes a pressurant tank; modelled with lumped mass not providing correct moment of inertia. Identifying issues such as these with the FE modelling is essential in order to have confidence that the FEM is reliable for further analyses. This serves to underline how essential it is to apply correlation techniques which are meaningful and understood rather than focus on meeting arbitrary criteria through means which may not be representative - such as has been demonstrated for the use of Guyan reduction on applications such as those considered in this work.

\section{Conclusion}

In this work, the traditional Guyan reduction method has been compared with a modal SEREP approach for FEMs of two spacecraft; BepiColombo and Aeolus. For the original test MPP DoFs, the Guyan reduced models matched poorly with the full models; only achieving MAC in excess of 0.9 for approximately $10 \%$ of the selected target modes for both spacecraft. When a kinetic energy approach was adopted to obtain a new sensor set (with the same number of DoFs as the original MPPs) this percentage increased to over $23 \%$ for BepiColombo and $44 \%$ for Aeolus. As such, it has been shown that modal KE based sensor set selection has the potential to slightly improve the quality of results from Guyan reduction. It is, however, important to note that there is still a significant difference in the results between full and Guyan reduced models. This discrepancy between full and Guyan reduced models may undermine the use of Guyan TAMs in COCs intended to assess the level of correlation between the test structure dynamics and those of the full FEM.

To further assess the influence of sensor placement on reduced model quality, the original MPPs for both spacecraft have been treated as candidate sets and smaller sets, half the size, have been selected by effective independence: an optimized set (selected from the MPP based on independence), and a 'worst case' set containing the remaining half of the MPP (omitted during optimization for displaying poor effective independence). The AutoMAC checks showed significantly lower off-diagonals with the selected DoFs than with those eliminated in the effective independence selection. The Guyan reduction based orthogonality check off-diagonal values were also 
markedly lower for the effective independence selected set than for the 'worst case' omitted set. Although the improvement was slightly less pronounced for the leading diagonal values, it is nevertheless evident that effective independence has potential to be a useful tool in refining sensor sets.

The SEREP method is theoretically advantageous in that, regardless of the sensor placement, it inherently produces an 'accurate' TAM; in which the natural frequencies and mode shapes match exactly to those of the full FEM, and there is perfect orthogonality with the reduced mass matrix. The potential practical benefits of SEREP have been confirmed through the cross-orthogonality comparisons of FEM results with corresponding test derived mode shapes. For both of the structures considered it was evident that, when good sensor placement was employed, SEREP produced consistently higher leading diagonal cross-orthogonality values than the Guyan TAMs.

\section{Acknowledgments}

The authors wish to thank Airbus Defence and Space United Kingdom for providing the spacecraft finite element models and funding for this study.

\section{References}

[1] R. J. Allemang and D. L. Brown, "A correlation coefficient for modal vector analysis," in Proceedings of the 1st international modal analysis conference, 1982, pp. 110-116.

[2] J. J. Wijker, Mechanical vibrations in spacecraft design: Springer, 2004.

[3] R. J. Allemang, "The modal assurance criterion-twenty years of use and abuse," Sound and Vibration, vol. 37, pp. 1423, 2003.

[4] P. Avitabile, "Model reduction and model expansion and their applications-part 1 theory," in Proceedings of the Twenty-Third International Modal Analysis Conference, Orlando, FL, USA, 2005.

[5] D. C. Kammer, "Test-analysis model development using an exact modal reduction," International Journal of Analytical and Experimental Modal Analysis, vol. 2, pp. 174-179, 1987.

[6] E. J. Bergman, M. S. Allen, D. C. Kammer, and R. L. Mayes, "Probabilistic investigation of sensitivities of advanced test-analysis model correlation methods," Journal of Sound and Vibration, vol. 329, pp. 2516-2531, 2010.

[7] P. Koutsovasilis and M. Beitelschmidt, "Comparison of model reduction techniques for large mechanical systems," Multibody System Dynamics, vol. 20, pp. 111-128, 2008.

[8] Y.-T. Chung and S. S. Simonian, "Assessments of model correlation using dynamic reduction and static reduction," SAE Technical Paper1988. 
[9] A. M. Freed and C. C. Flanigan, "A comparison of test-analysis model reduction methods(mass and stiffness matrix reduction of finite element model of large aerospace structures)," in International Modal Analysis Conference, 8 th, Kissimmee, FL, 1990, pp. 1344-1351.

[10] C. C. Flanigan, "Model Reduction Using Guyan IRS and Dynamic Methods," in SPIE International Society for Optical Engineering, 1998, pp. 172-176.

[11] P. Avitabile, F. Pechinsky, and J. O'Callahan, "Study of modal vector correlation using various techniques for model reduction," in Proceedings of the International Modal Analysis Conference, 1992, pp. 572-572.

[12] M. I. Friswell, S. D. Garvey, and J. E. T. Penny, "Model reduction using dynamic and iterated IRS techniques," Journal of Sound and Vibration, vol. 186, pp. 311-323, 1995.

[13] J. Guyan, Robert, "Reduction of Mass and Stiffness Matrices," AIAA Journal, vol. 3, pp. 380-380, 8 Sept 19641964.

[14] J. C. O'Callahan, "A procedure for an improved reduced system (IRS) model," in Proceedings of the 7th International Modal Analysis Conference, 1989, pp. 17-21.

[15] M. Paz, "Dynamic condensation," AIAA Journal, vol. 22, pp. 724-727, 1984.

[16] J. O'Callahan, P. Avitabile, and R. Riemer, "System equivalent reduction expansion process (SEREP)," in Proceedings of the 7th international modal analysis conference, 1989, pp. 29-37.

[17] D. Kammer, "A hybrid approach to test-analysis-model development for large space structures," Journal of Vibration and Acoustics, vol. 113, pp. 325-332, 1991.

[18] M. C. Bampton and J. Craig, Roy R, "Coupling of substructures for dynamic analyses," AIAA Journal, vol. 6, pp. 1313$1319,1968$.

[19] "MSC NASTRAN," ed. California, USA: MacNeal-Schwendler Corporation (MSC) Software, 2013.

[20] G. S. Aglietti, S. J. I. Walker, and A. Kiley, "On the use of SEREP for satellite FEM validation," Engineering Computations, vol. 29, pp. 580-595, 2012.

[21] K. K. Sairajan and G. S. Aglietti, "Robustness of System Equivalent Reduction Expansion Process on Spacecraft Structure Model Validation," AIAA Journal, vol. 50, pp. 2376-2388, 2012/11/01 2012.

[22] D. Kientzy, M. Richardson, and K. Blakely, "Using finite element data to set up modal tests," Sound and Vibration Magazine, vol. 23, pp. 16-23, 1989.

[23] B. Jarvis, "Enhancements to modal testing using finite elements," in 9th Conference International Modal Analysis Conference (IMAC), 1991, pp. 402-408.

[24] F. Udwadia, "Methodology for Optimum Sensor Locations for Parameter Identification in Dynamic Systems," Journal of Engineering Mechanics, vol. 120, pp. 368-390, 1994. 
[25] T. V. Langenhove and M. Brughmans, "Using MSC/NASTRAN and LMS/PRETEST to Find an Optimal Sensor Placement for Modal Identification and Correlation of Aerospace Structures," in LMS International, 1999.

[26] C. Papadimitriou, "Optimal sensor placement methodology for parametric identification of structural systems," Journal of Sound and Vibration, vol. 278, pp. 923-947, 2004.

[27] D. C. Kammer, "Sensor set expansion for modal vibration testing," Mechanical Systems and Signal Processing, vol. 19, pp. 700-713, 2005.

[28] D. C. Kammer, "Sensor placement for on-orbit modal identification and correlation of large space structures," Journal of Guidance, Control, and Dynamics, vol. 14, pp. 251-259, 1991.

[29] M. Salama, T. Rose, and J. Garba, "Optimal placement of excitations and sensors for verification of large dynamical systems," in Proceedings of the 28th Structures, Structural Dynamics, and Materials Conference, 1987, pp. 6-8.

[30] D. C. Kammer and M. L. Tinker, "Optimal placement of triaxial accelerometers for modal vibration tests," Mechanical Systems and Signal Processing, vol. 18, pp. 29-41, 2004.

[31] M. N. Helfrick, C. Niezrecki, P. Avitabile, and T. Schmidt, "3D digital image correlation methods for full-field vibration measurement," Mechanical Systems and Signal Processing, vol. 25, pp. 917-927, 2011.

[32] T. Chu, W. Ranson, and M. Sutton, "Applications of digital-image-correlation techniques to experimental mechanics," Experimental mechanics, vol. 25, pp. 232-244, 1985.

[33] T. Siebert, R. Wood, and K. Splitthof, "High speed image correlation for vibration analysis," in Journal of Physics: Conference Series, 2009, p. 012064.

[34] A. B. Stanbridge and D. J. Ewins, "Modal Testing Using a Scanning Laser Doppler Vibrometer," Mechanical Systems and Signal Processing, vol. 13, pp. 255-270, 1999.

[35] A. B. Stanbridge, M. Martarelli, and D. J. Ewins, "Measuring area vibration mode shapes with a continuous-scan LDV," Measurement, vol. 35, pp. 181-189, 2004.

[36] C. Warren, C. Niezrecki, P. Avitabile, and P. Pingle, "Comparison of FRF measurements and mode shapes determined using optically image based, laser, and accelerometer measurements," Mechanical Systems and Signal Processing, vol. 25, pp. 2191-2202, 2011.

[37] W. Wang, J. E. Mottershead, and C. Mares, "Vibration mode shape recognition using image processing," Journal of Sound and Vibration, vol. 326, pp. 909-938, 2009.

[38] W. Wang, J. E. Mottershead, A. Ihle, T. Siebert, and H. Reinhard Schubach, "Finite element model updating from fullfield vibration measurement using digital image correlation," Journal of Sound and Vibration, vol. 330, pp. 1599-1620, 2011. 
[39] D. Wang, F. A. DiazDelaO, W. Wang, X. Lin, E. A. Patterson, and J. E. Mottershead, "Uncertainty quantification in DIC with Kriging regression," Optics and Lasers in Engineering, vol. 78, pp. 182-195, 2016.

[40] Y. Y. Li, L. Cheng, L. H. Yam, and W. O. Wong, "Identification of damage locations for plate-like structures using damage sensitive indices: strain modal approach," Computers \& Structures, vol. 80, pp. 1881-1894, 2002.

[41] L.-H. Kang, D.-K. Kim, and J.-H. Han, "Estimation of dynamic structural displacements using fiber Bragg grating strain sensors," Journal of Sound and Vibration, vol. 305, pp. 534-542, 2007.

[42] F. dos Santos, B. Peeters, J. Lau, W. Desmet, and L. Góes, "An overview of experimental strain-based modal analysis methods," in Proceedings of the International Conference on Noise and Vibration Engineering (ISMA), Leuven, Belgium, 2014.

[43] ESA-ESTEC, "ECSS-E-ST-32-11 Modal Survey Assessment," ed. Noordwijk, The Netherlands: European Cooperation For Space Standardization, 2008, p. 78.

[44] NASA, "NASA-STD-5002 Load Analyses of Spacecraft and Payloads," in NASA Technical Standard, ed: National Aeronautics and Space Administration, 1996, p. 20.

[45] N. Popplewell, A. Bertels, and B. Arya, "A critical appraisal of the elimination technique," Journal of Sound and Vibration, vol. 31, pp. 213-233, 1973.

[46] G. R. Parker, T. L. Rose, and J. J. Brown, "Kinetic Energy Calculation as an Aid to Instrumentation Location in Modal Testing," in 1990 MSC World Users Conference, 1990.

[47] Z. Qureshi, T. Ng, and G. Goodwin, "Optimum experimental design for identification of distributed parameter systems," International Journal of Control, vol. 31, pp. 21-29, 1980.

[48] D. Ewins, Modal Testing: theory, practice and application 2nd Edition ed. Herts, UK: Research Studies Press Ltd., 2000.

[49] M. Friswell and J. E. Mottershead, Finite Element Model Updating in Structural Dynamics vol. 38: Springer, 1995.

[50] W. Heylen, S. Lammens, and P. Sas, Modal Analysis Theory and Testing: Katholieke Universiteit Leuven, Faculty of Engineering, Department of Mechanical Engineering, Division of Production Engineering, Machine Design and Automation, 2013.

[51] E. S. Agency. (2015, 02/02/2015). http://www.esa.int/spaceinimages/Images. Available: http://www.esa.int/spaceinimages/Images

[52] Y. T. Chung and M. L. Sernaker, "Assessment Target Mode Selection Criteria Payload Modal Survey," presented at the 12th International Modal Analysis Conference, 1994. 
[53] J. F. Mercer, G. S. Aglietti, and A. M. Kiley, "Modal and Frequency Domain Based Techniques for Finite Element Model Correlation," presented at the COMPDYN 2015 5th ECCOMAS Thematic Conference on Computational Methods in Structural Dynamics and Earthquake Engineering, Crete Island, Greece, 2015.

[54] P. Guillaume, P. Verboven, S. Vanlanduit, H. Van Der Auweraer, and B. Peeters, "A poly-reference implementation of the least-squares complex frequency-domain estimator," in Proceedings of IMAC, 2003, pp. 183-192.

[55] J. F. Mercer, A. M. Kiley, and G. S. Aglietti, "BepiColombo: sine test FEM correlation experiences," presented at the ISMA2014, Leuven, Belgium, 2014. 